\title{
Displacement and stress monitoring of a Panamax containership using inverse finite element method
}

\author{
Adnan Kefal*, Erkan Oterkus** \\ * Department of Naval Architecture, Ocean and Marine Engineering University of Strathclyde \\ 100 Montrose Street Glasgow G4 0LZ, United Kingdom \\ Phone: +44 (0)141 5484094 \\ e-mail: adnan.kefal@strath.ac.uk \\ ** Department of Naval Architecture, Ocean and Marine Engineering University of Strathclyde \\ 100 Montrose Street Glasgow G4 0LZ, United Kingdom \\ Phone: +44 (0)141 5483876 \\ e-mail: erkan.oterkus@strath.ac.uk
}

\begin{abstract}
The inverse Finite Element Method (iFEM) is a revolutionary methodology for realtime reconstruction of full-field structural displacements and stresses in plate and shell structures that are instrumented by strain sensors. This inverse problem is essential for structural health monitoring systems and commonly referred as 'displacement and stress monitoring' or 'shapeand stress-sensing'. In this study, displacement and stress monitoring of a Panamax containership is performed based on the iFEM methodology. A simple, efficient, and practically useful fournode quadrilateral inverse-shell element, iQS4, is used for the numerical implementation of the iFEM algorithm. Hydrodynamic analysis of the containership is performed for beam sea waves in order to calculate vertical and horizontal wave bending moments, and torsional wave moments acting on parallel mid-body of the containership. Several direct FEM analyses of the parallel mid-body are performed using the hydrodynamic wave bending and torsion moments. Then, experimentally measured strains are simulated by strains obtained from high-fidelity finite element solutions. After that, three different iFEM case studies of the parallel mid-body are performed utilizing the simulated sensor strains. Finally, the effect of sensor locations and number of sensors are assessed with respect to the solution accuracy.
\end{abstract}

Keywords: Displacement and Stress Monitoring, Structural Health Monitoring, Inverse Finite Element Method (iFEM), Panamax Containership. 


\section{Introduction}

Vessels are operated under challenging conditions because marine environment can cause failure of the structure due to extreme or cyclic loadings, corrosion, and erosion. Structural failure may lead to major accidents that may result in crew or passenger life loses, pollution of the marine environment, and very expensive maintenance/repair costs. Therefore, it is necessary to ensure safety, reliability, and integrity of ship structures for avoiding major accidents. Moreover, the number of new vessels is increasing day-to-day. Therefore, new structural designs, new construction techniques, and new materials are progressively being used in the shipbuilding industry. As a result, it is necessary to increase knowledge about the on-site structural performance not only for traditionally designed ships, but also for newly designed ships.

Structural Health Monitoring (SHM) is an interdisciplinary procedure that (1) integrates sensing systems to the structure, (2) processes the data collected from the sensing systems in real-time, and (3) provides decisive real-time information from the structure about its global and/or local structural state. Therefore, the necessities mentioned earlier and detailed structural management of the ships including inspection, maintenance, and repair plans can only be successfully accomplished if an application of SHM system is installed into the ships. In 1994, the International Maritime Organization (IMO) originally recommended the utilization of hull stress monitoring systems to facilitate the safe operation of ships. Then, the requirements for a typical hull structural monitoring system are regulated by class societies including ABS (1995), LR (2004), and DNV (2011). Recently, ABS (2015) has published a guide that discusses the need for fitting of hull condition monitoring systems on all types and sizes of merchant vessels. Besides, plenty of researchers considered the hull structural monitoring as an important case study. For example, different types of hull structural monitoring systems are investigated for various ship types (Van der Cammen 2008, Torkildsen et al. 2005, Andersson et al. 2011, Sielski 2012, Zhu and Frangopol 2013, Hageman et al. 2013, and Majewska et al. 2014). In particular, Phelps and Morris (2013) presented a general review regarding the technical aspects of available hull structural monitoring systems. However, most of the aforementioned SHM approaches don't take into account the advanced structural topologies and boundary conditions. Moreover, they mostly require sufficiently accurate loading information even though it is not easy to estimate 
dynamic loads of waves and winds due to the complexity and statistical feature of oceanographic phenomena. Furthermore, some of them are not appropriate for use in real-time due to the timeconsuming analysis.

Real-time processing of the data obtained from a system composed of a network of strain sensors is the key component of the SHM procedure for supplying trustworthy information about the structural condition. In other words, real-time reconstruction of three-dimensional structural displacements and stresses by utilizing discrete on-board strain measurements, known as 'displacement and stress monitoring' or 'shape- and stress-sensing', is the primary technology for performing an accurate SHM. Apart from the SHM systems mentioned earlier, Tessler and Spangler (2003, 2005) developed a powerful algorithm called inverse Finite Element Method (iFEM) for the purpose of displacement and stress monitoring of engineering structures. In contrast to other developed SHM methods, iFEM framework has a general applicability to any type of structural topology and boundary conditions because using inverse beam, frame, plate, and shell elements enables an effective discretization of the physical domain. The iFEM algorithm minimizes a weighted-least-squares functional with respect to nodal displacements in order to perform the shape-sensing of the structure. Since the iFEM weighted-least-squares functional is defined by using only strain-displacement relationship, the iFEM methodology can reconstruct the structural deformed shapes of the structure without prior knowledge of material properties and loading information. Therefore, unlike other proposed SHM systems, stability and accuracy of the shape-sensing results obtained through iFEM methodology is independent from material properties of the structure and any type of static or dynamic loadings acting on the structure. In fact, this special feature makes the iFEM methodology much more powerful than the other SHM systems. Once the structural deformed shape is obtained, the full field strains can be calculated from strain-displacement relationship. Then, full field stresses of the structure can be evaluated by using full field strains and material properties of the structure. This stress calculation not only allows iFEM methodology to perform stress-sensing of the structure, but also allows real-time damage predictions if the full field structural stresses are converted to an equivalent stress by using an appropriate failure criterion such as von Mises yield criterion.

Many different numerical and experimental iFEM studies have proved that the iFEM framework is an accurate, robust, and fast shape- and stress-sensing algorithm. For example, 
Tessler and Spangler (2004) developed a three-node inverse shell element (iMIN3) by using lowest-order anisoparametric $\mathrm{C}^{0}$ continuous functions and adopting kinematic assumptions of the first-order and shear-deformation theory. Tessler and Spangler (2004) numerically verified the precision of iMIN3 element for performing iFEM analysis of plate/shell structures. Also, Quach et al. (2005) and Vazquez et al. (2005) confirmed the robustness of the iMIN3 element by conducting laboratory tests that uses experimentally measured real-time strain data. Moreover, Tessler et al. (2012) enhanced iMIN3 element for displacement and stress monitoring of plate and shell structures undergoing large displacements. Apart from iMIN3, Kefal et al. (2016) have recently formulated a four-node quadrilateral inverse-shell element, iQS4, utilizing the kinematic assumptions of the first-order and transverse-shear deformation theory. This new element includes hierarchical drilling rotation degrees-of-freedom (DOF) and further extends the practical usefulness of iFEM for shape-sensing analysis of large-scale structures. Kefal et al. (2016) numerically verified the precision of the iQS4 element by the analysis of several validation and demonstration problems. Furthermore, Cerracchio et al. (2010) and Gherlone et al. $(2011,2012,2014)$ formulated a robust inverse frame element that uses kinematic assumptions of Timoshenko beam theory including stretching, bending, transverse-shear and torsion deformation modes. They numerically and experimentally validated capability of their inverse frame element by conducting several shape-sensing analyses of three-dimensional frame structures undergoing static and/or damped harmonic excitations. Moreover, Cerracchio et al. (2013, 2015a) has recently improved the original iFEM formulation of Tessler and Spangler $(2003,2005)$ by adding the kinematic assumptions of recently developed Refined Zigzag Theory (Tessler et al., 2009, 2010) in order to perform SHM of multi-layered composite and sandwich structures. The application of iFEM methodology for SHM of future aerospace vehicles is discussed by Tessler (2007) and Tessler et al. (2011). Likewise, another application of iFEM algorithm to real-time displacement monitoring of a composite stiffened panel is presented by Cerracchio et. al. (2015b). Apart from the aerospace applications, Kefal and Oterkus (2015) performed shape-sensing of a longitudinally and transversely stiffened plate as a fundamental application of the iFEM framework to SHM of marine structures. Similarly, Kefal and Oterkus (2016) presented a more sophisticated application of iFEM to marine structures namely displacement and stress monitoring of a chemical tanker based on iFEM algorithm. They performed iFEM case studies when the chemical tanker is subjected to the head sea waves 
because this phenomenon of ship advancing in waves can be very crucial for closed-decked ships such as chemical tanker. However, for open-decked ships such as containerships, head sea wave loads may be less important than beam sea wave loads due to torsional and warping stresses induced by torsional moments. Since hull girder torsion loading on containerships represent a major loading quantity particularly when combined with hull girder vertical and horizontal bending loads, displacement and stresses monitoring of a containership floating in beam sea waves should be investigated in addition to all proposed iFEM marine structure applications.

Hence, the main focus of this study is to demonstrate the application of the iFEM methodology for monitoring multi-axial deformations and stresses of a Panamax containership for the first time in the literature. For this purpose, the numerical implementation of the iFEM algorithm is done using the simple, efficient, and practically useful iQS4 element developed by Kefal et.al (2016). Hull form of a Panamax containership is designed performing several hull surface transformations of S175 containership. A typical mid-ship section is designed for the Panamax containership and the parallel mid-body of the containership is modelled using the iQS4 element. The 'smart methodology' proposed by Kefal et al. (2015) is followed to determine the optimum sensor locations for performing iFEM analyses of the parallel mid-body. First of all, hydrodynamic analysis of the containership is performed for beam sea waves. As an output, oscillatory pressures acting on the hydrodynamic panels, rigid body motions of the containership, and hydrodynamic section forces including vertical and horizontal bending moments and torsional moments are obtained. Secondly, several direct FEM analyses of the parallel mid-body are performed using the hydrodynamic wave bending and torsion moments. Then, experimentally measured strains are simulated by strains obtained from high-fidelity finite element solutions. Thirdly, three different iFEM analyses of the parallel mid-body are performed utilizing the simulated sensor strains obtained for three different cases. These are (1) pure vertical bending case, (2) pure horizontal bending case and (3) pure torsion case. Finally, the effect of sensor locations and number of sensors are assessed with respect to the solution accuracy of each iFEM analysis. 


\section{Inverse finite element formulation for shells (Kefal et al., 2016)}

\subsection{Quadrilateral inverse-shell element}

The four-node quadrilateral inverse-shell element (iQS4), developed by Kefal et al. (2016), is adopted to demonstrate the iFEM formulation for shells. The iQS4 element has six displacement DOF per node including a hierarchical drilling rotation (Fig. 1). As a result of the inclusion of drilling rotations, the iQS4 element has two beneficial features: (1) singular solutions can be simply avoided when modelling complex shell structures, (2) for membrane problems, iQS4 element has less tendency toward shear locking. In fact, these features make the iQS4 an efficient and practically useful element.

First of all, a set of convenient coordinate frames of reference are defined to guarantee the geometric uniqueness of the assembled shell structure. A local coordinate system $(x, y, z)$ serves as the element frame of reference, with its origin $(0,0,0)$ located at the centroid of mid-plane quadrilateral. It is assumed that the shell element has a uniform thickness $2 h$, and that $z \in[-h,+h]$ defines the thickness coordinate (Fig. 1). Herein, displacement, strain, and stress field of the iQS4 element is formulated using the local coordinate system. To assemble element matrices into a global system of equations, suitable transformation matrices defining the local to global transformations can easily be established with the element nodes referred to the global coordinates $(X, Y, Z)$. Details of how to allocate the local coordinate system at the centroid of mid-plane quadrilateral and establish the transformation matrices defining the local to global transformations are summarized in Appendix A.

The nodal DOF, consisting of positive $x$ translations $u_{i}$, positive $y$ translations $v_{i}$, and positive clockwise drilling rotations $\theta_{z i}$, define the $u$ and $v$ membrane displacements as

$$
\begin{aligned}
& u(x, y) \equiv u=\sum_{i=1}^{4} N_{i} u_{i}+\sum_{i=1}^{4} L_{i} \theta_{z i} \\
& v(x, y) \equiv v=\sum_{i=1}^{4} N_{i} v_{i}+\sum_{i=1}^{4} M_{i} \theta_{z i}
\end{aligned}
$$


The DOF of positive $z$ translation $w_{i}$ and positive counter clockwise rotations around the $\mathrm{x}$ - and y-axes, $\theta_{x i}$ and $\theta_{y i}$, define the transverse displacement $w$ and two bending rotations $\theta_{x}$ and $\theta_{y}$ by

$w(x, y) \equiv w=\sum_{i=1}^{4} N_{i} w_{i}-\sum_{i=1}^{4} L_{i} \theta_{x i}-\sum_{i=1}^{4} M_{i} \theta_{y i}$

$\theta_{x}(x, y) \equiv \theta_{x}=\sum_{i=1}^{4} N_{i} \theta_{x i}$

$\theta_{y}(x, y) \equiv \theta_{y}=\sum_{i=1}^{4} N_{i} \theta_{y i}$

where $N_{i}$ is bilinear isoparametric shape functions, $L_{i}$ and $M_{i}$ are the anisoparametric shape functions. These shape functions are analogous to four-node flat shell element described by Cook (1994) and MIN4 (Mindlin-type, four-nodes) element provided by Tessler and Hughes (1983).

The three components of the displacement vector of any material point within the element can be described as:

$u_{x}(x, y, z) \equiv u_{x}=u+z \theta_{y}$

$u_{y}(x, y, z) \equiv u_{y}=v-z \theta_{x}$

$u_{z}(x, y, z) \equiv u_{z}=w$

where $u_{x}$ and $u_{y}$ are the in-plane displacements and $u_{z}$ is the transverse displacement (deflection) across the uniform shell thickness.

For brevity, linear strain-displacement relations of linear elasticity are expressed in terms of nodal displacement vector, $\mathbf{u}^{e}$, as

$\boldsymbol{\varepsilon}_{b}=\mathbf{e}\left(\mathbf{u}^{e}\right)+z \mathbf{k}\left(\mathbf{u}^{e}\right)=\mathbf{B}^{m} \mathbf{u}^{e}+z \mathbf{B}^{k} \mathbf{u}^{e}$

$\boldsymbol{\varepsilon}_{s}=\mathbf{g}\left(\mathbf{u}^{e}\right)=\mathbf{B}^{s} \mathbf{u}^{e}$

with

$\boldsymbol{\varepsilon}_{b}=\left[\begin{array}{lll}\varepsilon_{x x} & \varepsilon_{y y} & \gamma_{x y}\end{array}\right]^{T}$

$\boldsymbol{\varepsilon}_{s}=\left[\begin{array}{ll}\gamma_{x z} & \gamma_{y z}\end{array}\right]^{T}$ 
and

$$
\begin{aligned}
\mathbf{u}^{e} & =\left[\begin{array}{llll}
\mathbf{u}_{1}^{e} & \mathbf{u}_{2}^{e} & \mathbf{u}_{3}^{e} & \mathbf{u}_{4}^{e}
\end{array}\right]^{T} \\
\mathbf{u}_{i}^{e} & =\left[\begin{array}{llllll}
u_{i} & v_{i} & w_{i} & \theta_{x i} & \theta_{y i} & \theta_{z i}
\end{array}\right]^{T} \operatorname{with}(i=1,2,3,4)
\end{aligned}
$$

where the matrices $\mathbf{B}^{m}, \mathbf{B}^{k}$, and $\mathbf{B}^{s}$ contain derivatives of the shape functions. The explicit forms of the shape functions $N_{i}, L_{i}$, and $M_{i}$, and $\mathbf{B}^{m}, \mathbf{B}^{k}$, and $\mathbf{B}^{s}$ matrices are given in Appendix B.

The membrane strains $\mathbf{e}\left(\mathbf{u}^{e}\right)$ are associated with the stretching of the middle surface. Therefore, $\mathbf{B}^{m}$ matrix contains the derivatives of the shape functions associated with the membrane behaviour. Furthermore, $\mathbf{k}\left(\mathbf{u}^{e}\right)$ and $\mathbf{g}\left(\mathbf{u}^{e}\right)$ are the bending curvatures and the transverse shear strains, respectively. Hence, $\mathbf{B}^{k}$ and $\mathbf{B}^{s}$ matrices contain the corresponding derivatives of shape functions used to define bending behaviour of the element. Note that the plane-stress assumption $\sigma_{z z}=0$ within the theory implies that the transverse-normal strain $\varepsilon_{z z}$ does not contribute to the strain energy.

\subsection{Input data from in-situ strain sensors}

Discrete in-situ strain measurements obtained from on-board sensors are crucial for the iFEM methodology. Conventional strain rosettes or embedded fibre-optic sensor networks such as Fibre Bragg Grating (FBG) sensors and Sensing Fibre Optic Cables can be used to collect large amount of on-board strain data. With today's technology, most of the strain sensors potentially offer a number of advantages for installation as they are lightweight, high speed, and not affected by electromagnetic interference and do not require re-calibration once installed. In order to calculate membrane and bending section strains experimentally, the in-situ strain rosettes should be located on top and bottom surfaces of the iQS4 element as shown in Fig. 2.

The experimentally measured (in-situ) membrane section strains $\mathbf{e}_{i}^{\varepsilon}$ and curvatures $\mathbf{k}_{i}^{\varepsilon}$ that correspond to their analytic counterparts, $\mathbf{e}\left(\mathbf{u}^{e}\right)$ and $\mathbf{k}\left(\mathbf{u}^{e}\right)$ given by Eq. (4), can be determined from the measured surface strains at $n$ discrete locations $\left(\mathbf{x}_{i}=x_{i}, y_{i}, \pm h\right) \quad(i=1, \ldots, n)$ located 
within the element. These in-situ section strains are computed as follows (Tessler and Spangler, 2005)

$\mathbf{e}_{i}^{\varepsilon}=\frac{1}{2}\left(\boldsymbol{\varepsilon}_{i}^{+}+\boldsymbol{\varepsilon}_{i}^{-}\right)$

$\mathbf{k}_{i}^{\varepsilon}=\frac{1}{2 h}\left(\boldsymbol{\varepsilon}_{i}^{+}-\boldsymbol{\varepsilon}_{i}^{-}\right)$

where

$\boldsymbol{\varepsilon}_{i}^{+}=\left[\begin{array}{lll}\varepsilon_{x x}^{+} & \varepsilon_{y y}^{+} & \gamma_{x y}^{+}\end{array}\right]_{i}^{T}$

and

$\boldsymbol{\varepsilon}_{i}^{-}=\left[\begin{array}{lll}\varepsilon_{x x}^{-} & \varepsilon_{y y}^{-} & \gamma_{x y}^{-}\end{array}\right]_{i}^{T}$

are the measured surface strains, with the superscripts ' + ' and '-' denoting the quantities that correspond to the top and bottom surface locations, respectively.

Although the experimentally measured surface strains can be used to compute the in-situ membrane strains $\mathbf{e}_{i}^{\varepsilon}$ and bending curvatures $\mathbf{k}_{i}^{\varepsilon}$, they cannot be directly used to calculate the insitu transverse shear strains $\mathbf{g}_{i}^{\varepsilon}$. A smoothing procedure, called the Smoothing Element Analysis (Tessler et al. 1998, 1999), enables the first-order derivatives of $\mathbf{k}_{i}^{\varepsilon}$ to be accurately computed and subsequently used to obtain the transverse shear strains $\mathbf{g}_{i}^{\varepsilon}$. It is noted, however, that in the deformation of thin shells, the contributions of $\mathbf{g}_{i}^{\varepsilon}$ are much smaller compared to the bending curvatures $\mathbf{k}_{i}^{\varepsilon}$. Since most of the marine structures are generally suitable to be modelled by using thin shells, the $\mathbf{g}_{i}^{\varepsilon}$ contributions can be safely omitted in the iFEM formulation.

\subsection{Weighted least-squares functional of inverse finite element method}

The inverse finite element method (iFEM) reconstructs the deformed shape of a discretized structure by minimizing a weighted least-squares functional with respect to the nodal DOF of the entire discretization. For an individual inverse element, this functional, $\Phi_{e}\left(\mathbf{u}^{e}\right)$, accounts for the membrane, bending and transverse shear deformations and is expressed according to Tessler et al. (2011) by 
$\Phi_{e}\left(\mathbf{u}^{e}\right)=w_{e}\left\|\mathbf{e}\left(\mathbf{u}^{e}\right)-\mathbf{e}^{\varepsilon}\right\|^{2}+w_{k}\left\|\mathbf{k}\left(\mathbf{u}^{e}\right)-\mathbf{k}^{\varepsilon}\right\|^{2}+w_{g}\left\|\mathbf{g}\left(\mathbf{u}^{e}\right)-\mathbf{g}^{\varepsilon}\right\|^{2}$

The squared norms expressed in Eq. (6a) can be written in the form of the normalized Euclidean norms

$$
\begin{aligned}
& \left\|\mathbf{e}\left(\mathbf{u}^{e}\right)-\mathbf{e}^{\varepsilon}\right\|^{2}=\frac{1}{n} \iint_{A^{e}} \sum_{i=1}^{n}\left(\mathbf{e}\left(\mathbf{u}^{e}\right)_{i}-\mathbf{e}_{i}^{\varepsilon}\right)^{2} d x d y \\
& \left\|\mathbf{k}\left(\mathbf{u}^{e}\right)-\mathbf{k}^{\varepsilon}\right\|^{2}=\frac{(2 h)^{2}}{n} \iint_{A^{e}} \sum_{i=1}^{n}\left(\mathbf{k}\left(\mathbf{u}^{e}\right)_{i}-\mathbf{k}_{i}^{\varepsilon}\right)^{2} d x d y \\
& \left\|\mathbf{g}\left(\mathbf{u}^{e}\right)-\mathbf{g}^{\varepsilon}\right\|^{2}=\frac{1}{n} \iint_{A^{e}} \sum_{i=1}^{n}\left(\mathbf{g}\left(\mathbf{u}^{e}\right)_{i}-\mathbf{g}_{i}^{\varepsilon}\right)^{2} d x d y
\end{aligned}
$$

where $A^{e}$ represents the mid-plane area of the element. The weighting constants $w_{e}, w_{k}$, and $w_{g}$ in Eq. (6a) are positive valued and are associated with the individual section strains. They control the complete coherence between the analytic section strains and their experimentally measured values. Their proper usage is especially critical for the problems involving relatively few locations of strain gages. When every analytic section strain has a corresponding measured insitu value $\left(\mathbf{e}_{i}^{\varepsilon}, \mathbf{k}_{i}^{\varepsilon}\right.$, and $\mathbf{g}_{i}^{\varepsilon}$ ), the weighting constants are set as $w_{e}=w_{k}=w_{g}=1$ in Eqs. (6b-6d).

In the case of a missing in-situ strain component, the corresponding weighting constant is set to be small, e.g., $\alpha=10^{-5}$, and Eqs. (6b-d) take on the reduced form

$$
\begin{aligned}
& \left\|\mathbf{e}\left(\mathbf{u}^{e}\right)\right\|^{2}=\iint_{A^{e}} \mathbf{e}\left(\mathbf{u}^{e}\right)^{2} d x d y \text { with }\left(w_{e}=\alpha\right) \\
& \left\|\mathbf{k}\left(\mathbf{u}^{e}\right)\right\|^{2}=(2 h)^{2} \iint_{A^{e}} \mathbf{k}\left(\mathbf{u}^{e}\right)^{2} d x d y \text { with }\left(w_{k}=\alpha\right) \\
& \left\|\mathbf{g}\left(\mathbf{u}^{e}\right)\right\|^{2}=\iint_{A^{e}} \mathbf{g}\left(\mathbf{u}^{e}\right)^{2} d x d y \text { with }\left(w_{g}=\alpha\right)
\end{aligned}
$$

where implementation of Eqs. (7) is performed on the component-by-component basis.

Furthermore, iFEM also permits the use of 'strain-less' inverse elements - the type of elements that do not have any in-situ section-strain measurements. For these 'strain-less' elements, all squared norms in Eqs. (7) are multiplied by the small weighting constants $w_{e}=w_{k}=w_{g}=\alpha=10^{-5}$. Therefore, an iFEM discretization can have very sparse measured strain 
data, and yet the necessary interpolation connectivity can still be maintained between the elements that have strain-sensor data.

By virtue of these assumptions, all strain compatibility relations are explicitly satisfied so that Eq. (6a) can be minimized with respect to the nodal displacement DOF, giving rise to

$\frac{\partial \Phi_{e}\left(\mathbf{u}^{e}\right)}{\partial \mathbf{u}^{e}}=\mathbf{k}^{e} \mathbf{u}^{e}-\mathbf{f}^{e}=0$

or simply

$\mathbf{k}^{e} \mathbf{u}^{e}=\mathbf{f}^{e}$

where $\mathbf{k}^{e}$ is the element left-hand-side matrix, $\mathbf{f}^{e}$ is the element right-hand-side vector that is a function of the measured strain values, and $\mathbf{u}^{e}$ is the nodal displacement vector of the element.

The element $\mathbf{k}^{e}$ matrix can be explicitly written in terms of the $\mathbf{B}^{m}, \mathbf{B}^{k}$, and $\mathbf{B}^{s}$ matrices and their corresponding weighting constants $w_{e}, w_{k}$, and $w_{g}$, and is given by

$\mathbf{k}^{e}=\iint_{A^{e}}\left(w_{e}\left(\mathbf{B}^{m}\right)^{T} \mathbf{B}^{m}+w_{k}(2 h)^{2}\left(\mathbf{B}^{k}\right)^{T} \mathbf{B}^{k}+w_{g}\left(\mathbf{B}^{s}\right)^{T} \mathbf{B}^{s}\right) d x d y$

The $\mathbf{f}^{e}$ vector is a function of the number of strain sensors within the element as well as the measured section-strain values, and is given by

$\mathbf{f}^{e}=\frac{1}{n} \iint_{A^{e}} \sum_{i=1}^{n}\left(w_{e}\left(\mathbf{B}^{m}\right)^{T} \mathbf{e}_{i}^{\varepsilon}+w_{k}(2 h)^{2}\left(\mathbf{B}^{k}\right)^{T} \mathbf{k}_{i}^{\varepsilon}+w_{g}\left(\mathbf{B}^{s}\right)^{T} \mathbf{g}_{i}^{\varepsilon}\right) d x d y$

Once the element (local) matrix equations are established, the element contributions to the global linear equation system of the discretized structure can be performed as

$\mathbf{K U}=\mathbf{F}$

with

$$
\begin{aligned}
& \mathbf{K}=\sum_{e=1}^{n e l}\left(\mathbf{T}^{e}\right)^{T} \mathbf{k}^{e} \mathbf{T}^{e} \\
& \mathbf{F}=\sum_{e=1}^{n e l}\left(\mathbf{T}^{e}\right)^{T} \mathbf{f}^{e}
\end{aligned}
$$




$$
\mathbf{U}=\sum_{e=1}^{n e l}\left(\mathbf{T}^{e}\right)^{T} \mathbf{u}^{e}
$$

where $\mathbf{T}^{e}$ is the transformation matrix of the nodal DOF of an element from the local to the global coordinate system (explicitly given in Appendix A), $\mathbf{K}$ is the global left-hand-side matrix (symmetric matrix and independent of the measured strain values), $\mathbf{U}$ is the global nodal displacement vector, $\mathbf{F}$ is the global right-hand-side vector (function of the measured strain values), and the parameter nel stands for the total number of inverse finite elements.

The global left-hand-side matrix $\mathbf{K}$ includes the rigid body motion mode of the discretized structure. Therefore, it is a singular matrix. By prescribing problem-specific displacement boundary conditions, the resulting system of equations can be reduced from Eq. (9a) as

$$
\mathbf{K}_{R} \mathbf{U}_{R}=\mathbf{F}_{R}
$$

where $\mathbf{K}_{R}$ is a positive definite matrix (always non-singular), and thus it is invertible. The solution of Eq. (10) is very fast because the matrix $\mathbf{K}_{R}$ remains unchanged for a given distribution of strain sensors and its inverse should be calculated only once during the length of the real time monitoring process. However, the right-hand-side vector $\mathbf{F}_{R}$ is dependent on the discrete surface strain data obtained from in-situ strain sensors. Hence, it needs to be updated during any deformation cycle. The matrix-vector multiplication $\mathbf{K}_{R}^{-1} \mathbf{F}_{R}$ gives rise to the unknown DOF vector $\mathbf{U}_{R}$, which provides the deformed structural shape at any real-time. Finally, the global displacement response can be assessed by computing the total displacement as

$$
U_{T}=\sqrt{U^{2}+V^{2}+W^{2}}
$$

where $U, V$, and $W$ are the translations (displacements) along the global $X-, Y$-, and $Z$-axes, respectively.

By using the evaluated displacement values, the continuous strain field throughout the structure can be obtained. Furthermore, the constitutive relationship between stress and strain will allow determination of stress distribution. For instance, the constitutive relationship for isotropic materials can be defined based on the plane-stress assumption $\left(\sigma_{z z}=0\right)$ as

$$
\boldsymbol{\sigma}_{b}=\mathbf{D}_{b} \boldsymbol{\varepsilon}_{b}=\mathbf{D}_{b} \mathbf{B}^{m} \mathbf{u}^{e}+z \mathbf{D}_{b} \mathbf{B}^{k} \mathbf{u}^{e}
$$


$\boldsymbol{\sigma}_{s}=\mathbf{D}_{s} \boldsymbol{\varepsilon}_{s}=\mathbf{D}_{s} \mathbf{B}^{s} \mathbf{u}^{e}$

where

$\boldsymbol{\sigma}_{b}=\left[\begin{array}{lll}\sigma_{x x} & \sigma_{y y} & \sigma_{x y}\end{array}\right]^{T}$

$\boldsymbol{\sigma}_{s}=\left[\begin{array}{ll}\sigma_{x z} & \sigma_{y z}\end{array}\right]^{T}$

and

$\mathbf{D}_{b}=\frac{E}{1-v^{2}}\left[\begin{array}{ccc}1 & v & 0 \\ v & 1 & 0 \\ 0 & 0 & (1-v) / 2\end{array}\right]$

$\mathbf{D}_{s}=\frac{E}{2(1+v)}\left[\begin{array}{ll}1 & 0 \\ 0 & 1\end{array}\right]$

are the reduced form of general isotropic stiffness matrix, with $E$ and $v$ denoting elastic modulus and Poisson's ratio of the isotropic material, respectively. Once the stress components at any point inside the iQS4 element domain are calculated using Eqs. (12), transformation of the stress components from the local to the global coordinate system can be performed as

$\boldsymbol{\sigma}=(\mathbf{T})^{T} \boldsymbol{\sigma}^{e} \mathbf{T}$

where

$\boldsymbol{\sigma}^{e}=\left[\begin{array}{ccc}\sigma_{x x} & \sigma_{x y} & \sigma_{x z} \\ \sigma_{x y} & \sigma_{y y} & \sigma_{y z} \\ \sigma_{x z} & \sigma_{y z} & 0\end{array}\right]$

$\boldsymbol{\sigma}=\left[\begin{array}{lll}\sigma_{X X} & \sigma_{X Y} & \sigma_{X Z} \\ \sigma_{X Y} & \sigma_{Y Y} & \sigma_{Y Z} \\ \sigma_{X Z} & \sigma_{Y Z} & \sigma_{Z Z}\end{array}\right]$

and $\mathbf{T}$ is the stress transformation matrix of an element from the local to the global coordinate system which is explicitly given in Appendix A. Finally, a suitable failure criterion can be used for damage detection as part of the SHM process. For instance, three-dimensional global stress components can be converted to an equivalent stress using the von Mises failure criterion as 


$$
\sigma_{V M}=\frac{1}{\sqrt{2}} \sqrt{\left(\sigma_{X X}-\sigma_{Y Y}\right)^{2}+\left(\sigma_{X X}-\sigma_{Z Z}\right)^{2}+\left(\sigma_{Y Y}-\sigma_{Z Z}\right)^{2}+6\left(\sigma_{X Y}^{2}+\sigma_{X Z}^{2}+\sigma_{Y Z}^{2}\right)}
$$

\section{3. iFEM analysis of a Panamax containership}

\subsection{Panamax containership model}

The body plan of S175 containership, given by Wu and Hermundstad (2002), is used as a 'parent ship hull form' in order to design a Panamax containership. Firstly, the hull form of the Panamax containership is obtained performing several hull form transformations of the S175 containership. These transformations are (1) linearly scaling based upon the characteristic breadth, and (2) linearly lengthening the parallel mid-body. Once the design of the hull form is completed, a typical mid-ship section is also designed for the Panamax containership. Isometric view of the hull surface below the draft waterline and mid-ship section drawings are depicted in Figs. 3 and 4, respectively.

For clarity, only one global Cartesian coordinate system $(X, Y, Z)$ serves as containership frame of reference, with its origin $(0,0,0)$ located at the still waterline and aligned vertically with the ship's centre of gravity. The $X-, Y$-, and $Z$-axes of the coordinate system point out the bow, portside, and opposite direction of gravity, respectively. According to the global coordinate system, when the containership is loaded at its design draft, the containership has the general particulars as listed in Table 1.

Parallel mid-body of the containership is composed of three full and two half cargo holds and is defined over the domain $X \in[-51.6 \mathrm{~m},+51.6 \mathrm{~m}]$. Each ends of the cargo hold are separated with watertight bulkheads and each cargo hold is equally subdivided into two cargo compartments with a non-watertight bulkhead. Each cargo compartment has longitudinal space of $12.48 \mathrm{~m}$ because they are designed for stowing 2x20 foot long containers. During the design of the containership, longitudinal frame spacing methodology is adopted and each cargo compartment is supported with three transverse frames. Moreover, both watertight and nonwatertight transverse bulkheads have length of $1.56 \mathrm{~m}$ and each bulkhead is supported with a transverse frame at both ends. For simplicity, all the structural components including plates, stiffeners, and transverse frames have been designed to have the uniform thickness of $30 \mathrm{~mm}$ and they are made of steel having elastic modulus of $E=210 \mathrm{GPa}$ and Poisson's ratio of $v=0.3$. In 
order to represent the complexity of the structure more clearly, an isometric view of the parallel mid-body is illustrated in Fig. 5.

\subsection{Hydrodynamic-FEM analysis of the containership}

In this study, the above stated parallel mid-body is analysed using the iFEM/iQS4 methodology to monitor the multi-axial deformations and stresses of the Panamax containership. For each iFEM case study, optimum strain-sensor locations are determined utilizing 'the smart system/methodology' proposed by Kefal et. al. (2015). Firstly, hydrodynamic loads acting on the containership is calculated performing a hydrodynamic analysis. Secondly, an accurate reference solution is established performing a direct FEM analysis. Then, the FEM deflections and rotations are used to compute the simulated sensor strains. Finally, the iFEM analysis of the parallel mid-body is performed using the simulated sensor strains. In-house hydrodynamic and FEM codes developed by Kefal and Oterkus (2014) are used for the hydrodynamic-FEM analysis.

The total weight of the containership is distributed along the length between perpendiculars as depicted in Fig. 6. At this loading condition, the containership is assumed to float with zero forward speed in beam sea waves. Using a full hydrodynamic model consisted of 4,912 flat quadrilateral panels, the hydrodynamic analysis is performed. As a result, six DOF motions and hydrodynamic pressures of the containership are obtained for unit wave amplitude and wave frequencies ranging from $0.05 \mathrm{rad} / \mathrm{s}$ to $1.2 \mathrm{rad} / \mathrm{s}$. The nonzero rigid body motion amplitudes, namely sway, heave, and roll motions, are presented in Figs. 7 and 8, respectively.

At any section along the length, hydrodynamic section forces can be calculated using the six DOF motions, the hydrodynamic pressures, and the total weight distribution. For open-decked ships such as containerships, beam sea wave loads have a great impact on the strength of the structure due to torsional and warping stresses induced by torsional moments. As can be seen from Fig. 8, the roll motion amplitude significantly increases between wave frequencies of 0.2 $\mathrm{rad} / \mathrm{s}$ and $0.4 \mathrm{rad} / \mathrm{s}$. This result clearly demonstrates the wave frequency interval where the

torsional wave moments become the most critical loading component. Therefore, vertical and horizontal wave bending moments, $M_{y}$ and $M_{z}$, and torsional wave moments, $M_{x}$, at section 
$X=0 \mathrm{~m}$ are compared between the wave frequencies ranging from $0.2 \mathrm{rad} / \mathrm{s}$ to $0.4 \mathrm{rad} / \mathrm{s}$ as depicted in Fig. 9.

As can be seen from Fig. 9, $0.31 \mathrm{rad} / \mathrm{s}$ is the most critical wave frequency for performing the structural analysis, so that vertical and horizontal wave bending moments, $M_{y}=20686 \mathrm{kNm} / \mathrm{m}$ and $M_{z}=23261 \mathrm{kNm} / \mathrm{m}$, respectively, and torsional wave moment, $M_{x}=18446 \mathrm{kNm} / \mathrm{m}$, at 0.31 $\mathrm{rad} / \mathrm{s}$, are chosen as load input for the following direct FEM analysis. For clarity, total hydrodynamic pressures $P$ due to the oscillation at wave frequency of $0.31 \mathrm{rad} / \mathrm{s}$ are plotted over the underwater panels of the containership as depicted in Fig. 10.

Structural analysis of the parallel mid-body is performed for three different loading cases. They are (1) pure vertical bending case, (2) pure horizontal bending case and (3) pure torsion case. For each loading case, the suitable moment obtained from hydrodynamic analysis is uniformly applied along the length of the parallel mid-body. The resultant deformations due to these three loading scenarios may be symmetric/antisymmetric with respect to $X Z$ - and/or $Y Z$ planes. Moreover, geometry of the parallel mid-body is symmetric with respect to both $X Z$ - and YZ-planes. Therefore, only one-fourth of the mid-body is modelled instead of modelling the entire mid-body. The translations along the normal axis and the rotations around the in-plane axes are constrained for symmetry boundary condition, while the rotation around the normal axis and the translations along the in-plane axes are constrained for anti-symmetry boundary condition. For each loading case, the boundary conditions (BC) imposed to $X Z$ - and $Y Z$-planes are listed in Table 2. Once the boundary conditions are applied to the FEM model, an accurate reference solution is established performing a convergence study. The most refined mesh consists of 246,484 shell elements, possessing 1,428,738 DOF. After the execution of direct FEM analysis for each loading case, experimentally measured strains, $\boldsymbol{\varepsilon}_{i}^{+}$and $\boldsymbol{\varepsilon}_{i}^{-}$, are simulated by strains obtained from the FEM analyses.

\section{3. iFEM case studies of the containership}

Three different iFEM analyses of the parallel mid-body are performed utilizing the simulated sensor strains obtained for pure vertical bending case, pure horizontal bending case, and pure torsion case, respectively. A different strain-rosette network is used for each iFEM analysis of the mid-body. The resulting deformations of the mid-body exhibit both stretching and bending 
response due to the complexity of the structural topology. Hence, the strain-rosettes have to be placed on both the top and bottom surfaces of the plates. To take the advantage of the symmetry and remain consistent with the above stated boundary conditions, only one-fourth of parallel mid-body is discretised using iQS4 elements in the following iFEM analyses. Moreover, the hull structure is made up with thin shells so the weighting constants for transverse shear strains are set as $w_{g}=10^{-5}$ in the following iFEM analyses.

In the first case study, i.e. pure vertical bending case, the iQS4 model of the parallel mid-body has 15,318 elements with top- and bottom-surface strain rosettes located within 327 select elements as shown in Figs. 11 and 12. For an iQS4 element which has no in-situ strain components, the corresponding weighting coefficients are set to $10^{-5}$.

In Fig. 13, the contour plot for the $U_{T}$ displacement is depicted for the first iFEM analysis. Although only one-fourth of the mid-body is analysed using the iQS4 mesh presented in Figs. 11 and 12, the results are illustrated using an iQS4 mesh corresponds to the entire parallel mid-body for a better visualization of the deformed shape. Remarkably, the deformed shape of the midbody confirms the pure vertical bending of the structure (Fig. 13). To investigate the accuracy of iFEM predictions for the $U_{T}$ displacement, the percent difference (PD) between iFEM and direct FEM results for $U_{T}$ displacement can be computed for each node $i$ as

$$
P D\left(U_{T}\right)=\left|\frac{U_{T, i}^{i F E M}-U_{T, i}^{F E M}}{U_{T, \text { max }}^{F E M}}\right| \times 100 \%
$$

where $U_{T, i}^{i F E M}$ is iFEM prediction for the $U_{T}$ displacement at node $i, U_{T, i}^{F E M}$ is direct FEM prediction for the $U_{T}$ displacement at node $i$, and $U_{T, \max }^{F E M}$ is direct FEM prediction of the maximum $U_{T}$ displacement. In Fig. 14, contour plot of $P D\left(U_{T}\right)$ is shown for the first iFEM analysis. As can be seen from Fig. 14, the maximum $P D\left(U_{T}\right)$ is equal to $7.248 \%$ and the maximum $P D\left(U_{T}\right)$ is located at the node where the maximum $U_{T}$ displacement is occurred (Fig. 13). Therefore, this result clearly demonstrates the superior accuracy of the iFEM solutions for displacement monitoring. 
Once the structural deformed shape is obtained, the von Mises stresses, $\sigma_{V M}$, on the top surfaces of the shells are calculated. In Fig. 15, the contour plot for the $\sigma_{V M}$ stress is presented for the first iFEM analysis. Moreover, the accuracy of iFEM predictions for the $\sigma_{V M}$ stress is examined by calculating the percent difference between iFEM and direct FEM predictions for $\sigma_{V M}$ stress at each node $i$ as

$P D\left(\sigma_{V M}\right)=\left|\frac{\sigma_{V M, i}^{i F E M}-\sigma_{V M, i}^{F E M}}{\sigma_{V M, \text { max }}^{F E M}}\right| \times 100 \%$

where $\sigma_{V M, i}^{i F E M}$ is iFEM prediction for the $\sigma_{V M}$ stress at node $i, \sigma_{V M, i}^{F E M}$ is direct FEM prediction for the $\sigma_{V M}$ stress at node $i$, and $\sigma_{V M \text {, max }}^{F E M}$ is direct FEM prediction of the maximum $\sigma_{V M}$ stress. In Fig. 16, contour plot of $P D\left(\sigma_{V M}\right)$ is shown for the first iFEM analysis. The maximum $P D\left(\sigma_{V M}\right)$ is equal to $12.569 \%$ and its location is identical to the location of the node where the maximum $\sigma_{V M}$ stress is occurred (Figs. 15 and 16). Therefore, this result confirms superior precision of the iFEM solutions for stress monitoring. These results also confirm the strainsensor locations depicted in Figs. 11 and 12 are the optimum locations for performing an accurate shape- and stress-sensing of the Panamax containership subjected to vertical wave bending moment.

In the second case study, i.e. pure horizontal bending case, the iQS4 model of the parallel mid-body has 15,318 elements with top- and bottom-surface strain rosettes located within 413 select elements as shown in Figs. 17 and 18. For an iQS4 element which has no in-situ strain components, the corresponding weighting coefficients are set to $10^{-5}$.

In Fig. 19, the contour plot for the $U_{T}$ displacement is demonstrated for the second iFEM analysis. Similar to the first iFEM case study, a better visualization of the deformed shape is achieved using an iQS4 mesh corresponds to the entire parallel mid-body. As can be seen from the Fig. 19, the deformed shape of the mid-body is identical to the pure horizontal bending of the structure. To demonstrate the accuracy of iFEM predictions for the $U_{T}$ displacement, contour plot of $P D\left(U_{T}\right)$ for the second iFEM analysis is shown in Fig. 20. According to Figs. 19 and 20, $P D\left(U_{T}\right)$ is approximately equal to $5.5 \%$ at the location where the maximum $U_{T}$ 
displacement is occurred. Hence, this result clearly demonstrates remarkable precision of the iFEM solutions for shape-sensing.

In Fig. 21, the contour plot for the $\sigma_{V M}$ stress calculated at the top surfaces of the shells is presented for the second iFEM case study. Also, the accuracy of iFEM predictions for the $\sigma_{V M}$ stress is examined by presenting contour plot of $P D\left(\sigma_{V M}\right)$ as shown in Fig. 22. As can be seen from the Figs. 21 and 22, $P D\left(\sigma_{V M}\right)$ is around $8 \%$ at the location where the maximum $\sigma_{V M}$ stress is occurred. Consequently, this result confirms high precision of the iFEM solutions for stress-sensing. Furthermore, these results verify that the strain-sensor locations presented in Figs. 17 and 18 are the optimum locations for performing a precise displacement and stress monitoring of the Panamax containership exposed to horizontal wave bending moment.

In the third case study, i.e. pure torsion case, the iQS4 model of the parallel mid-body has 15,318 elements with top- and bottom-surface strain rosettes located within 442 select elements as shown in Figs. 23 and 24. For an iQS4 element which has no in-situ strain components, the corresponding weighting coefficients are set to $10^{-5}$.

In Fig. 25, the contour plot for the $U_{T}$ displacement is demonstrated for the third iFEM analysis. An iQS4 mesh that represents the entire parallel mid-body is utilized for a better visualization of the deformed shape. As can be seen from Fig. 25, the deformed shape of the mid-body exhibits the pure torsion of the structure. According to Figs. 13, 19, and 25, the maximum $U_{T}$ displacement induced due by torsional moment is much larger than the maximum $U_{T}$ displacement caused due to vertical and horizontal bending moments. This result proofs the significance of hull girder torsion loading on containerships floating in beam sea waves. In Fig. 26, contour plot of $P D\left(U_{T}\right)$ is depicted in order to investigate the accuracy of iFEM predictions for the $U_{T}$ displacement found in the third iFEM case study. As can be seen from Figs. 25 and 26, $P D\left(U_{T}\right)$ is approximately equal to $7.1 \%$ at the location where the maximum $U_{T}$ displacement is occurred. Hence, this result clearly indicates the significant precision of the iFEM solutions for displacement monitoring. 
In Fig. 27, the contour plot for the $\sigma_{V M}$ stress calculated at the top surfaces of the shells is presented for the third iFEM case study. Additionally, in order to examine $\sigma_{V M}$ stress found in the third iFEM case study, contour plot of $P D\left(\sigma_{V M}\right)$ is shown in Fig. 28. According to Figs. 27 and 28, $P D\left(\sigma_{V M}\right)$ is approximately $10.7 \%$ at the location where the maximum $\sigma_{V M}$ stress is occurred. Hence, this result proofs superior precision of the iFEM solutions for stress monitoring. These results also validate that the strain-sensor locations demonstrated in Figs. 23 and 24 are the optimum locations for performing a precise shape- and stress-sensing of the Panamax containership subjected to torsional wave moment. According to the results found in the three different iFEM case studies, it can be concluded that iFEM is a superior, powerful, and innovative technology for the structural health monitoring of marine structures.

\section{Concluding remarks}

Displacement and stress monitoring of a Panamax containership is accomplished based on iFEM methodology. The iFEM algorithm reconstructs the full-field displacements, strains, and stresses of a structure from discrete in-situ strain measurements. The iFEM formulation is based on a least-squares variational principle originally developed by Tessler and Spangler (2005). Herein, a recently developed four-node quadrilateral inverse-shell element (iQS4) is utilized for performing the shape- and stress-sensing of the containership. The parallel mid-body of the containership is modelled using iQS4 elements as a representative of the global structural model. Firstly, the hydrodynamic analysis of the Panamax containership is performed for the beam sea waves. Secondly, the direct FEM analysis of the mid-body is performed using the hydrodynamic wave bending and torsion moments as input. Then, the FEM deflections and rotations are used to compute the simulated sensor strains. Thirdly, three different iFEM analyses of the parallel midbody are performed utilizing the simulated sensor strains obtained for three different cases. These are (1) pure vertical bending case, (2) pure horizontal bending case and (3) pure torsion case. Based on the same iQS4 mesh, a different network of strain-sensors is proposed for each iFEM analysis. Then, the deformed shape and von Mises stresses of the containership are reconstructed using in-situ strain data obtained from each proposed network of strain-sensors. According to the accuracy of the displacement and stress results, the optimum strain-sensor locations are identified and clearly demonstrated for each iFEM case study. Hence, the numerical 
results confirmed the robustness of the iFEM methodology for monitoring multi-axial deformations and stresses of a Panamax containership floating in beam sea waves. 


\section{Appendix A}

The global coordinates of the iQS4 element nodes are given as

$$
\mathbf{X}_{i}=\left[\begin{array}{lll}
X_{i} & Y_{i} & Z_{i}
\end{array}\right]^{T}(i=1,2,3,4)
$$

Each edge length, $d_{i}$, and global coordinates of each edge mid-point, $\mathbf{c}_{i}$, can be calculated as

$\left.\begin{array}{l}d_{i}=\left\|\mathbf{X}_{j}-\mathbf{X}_{i}\right\| \\ \mathbf{c}_{i}=\frac{\mathbf{X}_{j}+\mathbf{X}_{i}}{2}\end{array}\right\}(i=1,2,3,4 ; j=2,3,4,1)$

Using Eq. (A2), global coordinates of mid-plane quadrilateral centroid can be defined as

$\mathbf{C}=\frac{\sum_{k=1}^{4} \mathbf{c}_{k} d_{k}}{\sum_{k=1}^{4} d_{k}}$

Unit normal vector to the mid-plane quadrilateral, $\mathbf{n}$, and unit vectors along local $y$ - and $x$-axis, $\mathbf{p}$ and $\mathbf{I}$, can respectively be computed as:

$$
\begin{aligned}
& \mathbf{n}=\frac{\mathbf{A} \times \mathbf{B}}{\|\mathbf{A} \times \mathbf{B}\|} \\
& \mathbf{p}=\frac{\mathbf{A}+\mathbf{B}}{\|\mathbf{A}+\mathbf{B}\|} \\
& \mathbf{l}=\mathbf{p} \times \mathbf{n}
\end{aligned}
$$

where

$$
\begin{aligned}
& \mathbf{A}=\mathbf{X}_{3}-\mathbf{X}_{1} \\
& \mathbf{B}=\mathbf{X}_{4}-\mathbf{X}_{2}
\end{aligned}
$$

are diagonal vectors, with A pointing out from node-1 to node-3, whereas B pointing out from node-2 to node-4. Using Eq. (A1), Eq. (A3), and Eqs. (A4b-c), local coordinates of the iQS4 element nodes can be determined as 
$\left.\begin{array}{l}x_{i}=\left(\mathbf{X}_{i}-\mathbf{C}\right) \cdot \mathbf{l} \\ y_{i}=\left(\mathbf{X}_{i}-\mathbf{C}\right) \cdot \mathbf{p}\end{array}\right\}(i=1,2,3,4)$

With the unit vectors $\mathbf{n}, \mathbf{p}$, and $\mathbf{l}$, given in Eqs.(4a-c), the transformation matrix of the nodal DOF of an element from the local to the global coordinate system can be defined as

$\mathbf{T}^{e}=\left[\begin{array}{llllllll}\mathbf{T} & 0 & 0 & 0 & 0 & 0 & 0 & 0 \\ 0 & \mathbf{T} & 0 & 0 & 0 & 0 & 0 & 0 \\ 0 & 0 & \mathbf{T} & 0 & 0 & 0 & 0 & 0 \\ 0 & 0 & 0 & \mathbf{T} & 0 & 0 & 0 & 0 \\ 0 & 0 & 0 & 0 & \mathbf{T} & 0 & 0 & 0 \\ 0 & 0 & 0 & 0 & 0 & \mathbf{T} & 0 & 0 \\ 0 & 0 & 0 & 0 & 0 & 0 & \mathbf{T} & 0 \\ 0 & 0 & 0 & 0 & 0 & 0 & 0 & \mathbf{T}\end{array}\right]$

(A.6a)

where

$$
\mathbf{T}=\left[\begin{array}{lll}
\mathbf{l}^{T} & \mathbf{p}^{T} & \mathbf{n}^{T}
\end{array}\right]^{T}
$$

is the stress transformation matrix from the local to the global coordinate system. 


\section{Appendix B}

The shape functions $N_{i}, L_{i}$, and $M_{i}$, which are used to describe both membrane and bending capability of the iQS4 element as given in Eqs. (1a-b) and Eqs. (2a-c), are respectively defined as

$$
\begin{aligned}
& N_{1}=\frac{(1-s)(1-t)}{4} \\
& N_{2}=\frac{(1+s)(1-t)}{4} \\
& N_{3}=\frac{(1+s)(1+t)}{4} \\
& N_{4}=\frac{(1-s)(1+t)}{4} \\
& N_{5}=\frac{\left(1-s^{2}\right)(1-t)}{16} \\
& N_{6}=\frac{(1+s)\left(1-t^{2}\right)}{16} \\
& N_{7}=\frac{\left(1-s^{2}\right)(1+t)}{16} \\
& N_{8}=\frac{(1-s)\left(1-t^{2}\right)}{16}
\end{aligned}
$$

and

$$
\begin{aligned}
& L_{1}=y_{14} N_{8}-y_{21} N_{5} \\
& L_{2}=y_{21} N_{5}-y_{32} N_{6} \\
& L_{3}=y_{32} N_{6}-y_{43} N_{7} \\
& L_{4}=y_{43} N_{7}-y_{14} N_{8} \\
& M_{1}=x_{41} N_{8}-x_{12} N_{5} \\
& M_{2}=x_{12} N_{5}-x_{23} N_{6} \\
& M_{3}=x_{23} N_{6}-x_{34} N_{7} \\
& M_{4}=x_{34} N_{7}-x_{41} N_{8}
\end{aligned}
$$

Note that $x_{i j}$ and $y_{i j}$ can be expressed in terms of local coordinates of iQS4 element as 
$\left.\begin{array}{l}x_{i j}=x_{i}-x_{j} \\ y_{i j}=y_{i}-y_{j}\end{array}\right\}(i=1,2,3,4 ; j=1,2,3,4)$

and the parent space coordinates are defined as $s, t \in[-1,+1]$.

The derivatives of shape functions $\mathbf{B}^{m}, \mathbf{B}^{k}, \mathbf{B}^{s}$ which are given in Eqs. (4a-b) are defined as

$\mathbf{B}^{m}=\left[\begin{array}{llll}\mathbf{B}_{1}^{m} & \mathbf{B}_{2}^{m} & \mathbf{B}_{3}^{m} & \mathbf{B}_{4}^{m}\end{array}\right]$

$\mathbf{B}^{k}=\left[\begin{array}{llll}\mathbf{B}_{1}^{k} & \mathbf{B}_{2}^{k} & \mathbf{B}_{3}^{k} & \mathbf{B}_{4}^{k}\end{array}\right]$

$\mathbf{B}^{s}=\left[\begin{array}{llll}\mathbf{B}_{1}^{s} & \mathbf{B}_{2}^{s} & \mathbf{B}_{3}^{s} & \mathbf{B}_{4}^{s}\end{array}\right]$

where

$$
\left.\begin{array}{rl}
\mathbf{B}_{i}^{m} & =\left[\begin{array}{cccccc}
N_{i, x} & 0 & 0 & 0 & 0 & L_{i, x} \\
0 & N_{i, \mathrm{y}} & 0 & 0 & 0 & M_{i, x} \\
N_{i, \mathrm{y}} & N_{i, x} & 0 & 0 & 0 & L_{i, \mathrm{y}}+M_{i, x}
\end{array}\right] \\
\mathbf{B}_{i}^{k} & =\left[\begin{array}{llllll}
0 & 0 & 0 & 0 & N_{i, x} & 0 \\
0 & 0 & 0 & -N_{i, \mathrm{y}} & 0 & 0 \\
0 & 0 & 0 & -N_{i, \mathrm{x}} & N_{i, \mathrm{y}} & 0
\end{array}\right] \\
\mathbf{B}_{i}^{s} & =\left[\begin{array}{llllll}
0 & 0 & N_{i, x} & -L_{i, x} & -M_{i, x}+N_{i} & 0 \\
0 & 0 & N_{i, \mathrm{y}} & -L_{i, \mathrm{y}}-N_{i} & -M_{i, \mathrm{y}} & 0
\end{array}\right]
\end{array}\right\}(i=1,2,3,4)
$$




\section{References}

American Bureau of Shipping. (1995). Guide for Hull Condition Monitoring Systems.

American Bureau of Shipping. (2015). Guide for Hull Condition Monitoring Systems.

Andersson, S., Haller, K., Hellbratt, S. E., \& Hedberg, C. (2011). Damage monitoring of ship FRP during exposure to explosion impacts. In Proc. 18th Inter. Conf. Composite Materials, Jeju Island, Korea.

Cerracchio, P., Gherlone, M., Mattone, M., Di Sciuva, M., \& Tessler, A. (2010). Shape sensing of three-dimensional frame structures using the inverse finite element method. In: Proceedings of 5th European Workshop on Structural Health Monitoring, Sorrento, Italy.

Cerracchio, P., Gherlone, M., Di Sciuva, M., \& Tessler, A. (2013). Shape and Stress Sensing of Multilayered Composite and Sandwich Structures Using an Inverse Finite Element Method. In: Proceedings of V International Conference on Computational Methods for Coupled Problems in Science and Engineering, Ibiza, Spain.

Cerracchio, P., Gherlone, M., Di Sciuva, M., \& Tessler, A. (2015a). A novel approach for displacement and stress monitoring of sandwich structures based on the inverse Finite Element Method. Composite Structures, 127, 69-76.

Cerracchio, P., Gherlone, M., \& Tessler A. (2015b). Real-time displacement monitoring of a composite stiffened panel subjected to mechanical and thermal loads. Meccanica, 50, 2487-2496.

Cook, R. D. (1994). Four-node 'flat' shell element: drilling degrees of freedom, membranebending coupling, warped geometry, and behaviour. Computers and Structures, 50(4), 549-555.

Det Norske Veritas, Hull Monitoring Systems. (2011). DNV Rules for Classification of Ships, Part 6, Chapter 11.

Gherlone, M., Cerracchio, P., Mattone, M., Di Sciuva, M., \& Tessler, A. (2011). Beam shape sensing using inverse finite element method: theory and experimental validation. In: Proceeding of 8th International Workshop on Structural Health Monitoring, Stanford, CA 
Gherlone, M., Cerracchio, P., Mattone, M., Di Sciuva, M., \& Tessler, A. (2012). Shape sensing of 3D frame structures using an inverse Finite Element Method. International Journal of Solids and Structures, 49(22), 3100-3112.

Gherlone, M., Cerracchio, P., Mattone, M., Di Sciuva, M., \& Tessler, A. (2014). An inverse Finite Element Method for beam shape sensing: theoretical framework and experimental validation. Smart Materials and Structures, 23(4), 045027.

Hageman, R., Aalbetrs, P., Shaik, M., \& Van den Boom, H. (2013). Development of an Advisory Hull Fatigue Monitoring System. In: Proceedings of SNAME Annual Meeting \& Expo \& Ship Production Symposium, Bellevue, WA.

Kefal, A., \& Oterkus, E. (2014). D3.3 (WP3) - Hydrodynamic and structural analysis. Public Deliverable, The INCASS Project (FP7/2007-2013 grant agreement no 605200).

Kefal, A., \& Oterkus, E. (2015). Structural Health Monitoring of marine structures by using inverse Finite Element Method. Analysis and Design of Marine Structures V, 341-349.

Kefal, A., Hizir, O., \& Oterkus, E. (2015). A smart system to determine sensor locations for structural health monitoring of ship structures. In: Proceedings of 9th International Workshop on Ship and Marine Hydrodynamics, Glasgow, UK, 2015.

Kefal, A., \& Oterkus, E. (2016). Displacement and stress monitoring of a chemical tanker based on inverse finite element method. Ocean Engineering, 112, 33-46.

Kefal, A., Oterkus, E., Tessler, A., \& Spangler, J.L. (2016). A Quadrilateral Inverse-Shell Element with Drilling Degrees of Freedom for Shape Sensing and Structural Health Monitoring. Engineering Science and Technology, an International Journal, In Press. http://dx.doi.org/10.1016/j.jestch.2016.03.006.

Lloyds Register. (2004). ShipRight Ship Event Analysis.

Majewska, K., Mieloszyk, M., Ostachowicz, W., \& Król, A. (2014). Experimental method of strain/stress measurements on tall sailing ships using Fibre Bragg Grating sensors. Applied Ocean Research, 47, 270-283. 
Phelps, B., \& Morris, B. (2013). Review of Hull Structural Monitoring Systems for Navy Ships (No. DSTO-TR-2818). Defense Science and Technology Organization Victoria (Australia) Maritime Platforms Div.

Quach, C.C., Vazquez, S.L., Tessler, A., Moore, J.P., Cooper, E.G., \& Spangler, J.L. (2005). Structural anomaly detection using fiber optic sensors and inverse finite element method. In: Proceedings of AIAA Guidance, Navigation, and Control Conference and Exhibit, San Francisco, California.

Sielski, R. A. (2012). Ship structural health monitoring research at the Office of Naval Research. JOM, 64(7), 823-827.

Tessler, A., \& Hughes, T. J. (1983). An improved treatment of transverse shear in the Mindlintype four-node quadrilateral element. Computer Methods in Applied Mechanics and Engineering, 39(3), 311-335.

Tessler, A., Riggs, H. R., Freese, C. E., \& Cook, G. M. (1998). An improved variational method for finite element stress recovery and a posteriori error estimation. Computer Methods in Applied Mechanics and Engineering, 155(1), 15-30.

Tessler, A., Riggs, H. R., \& Dambach, M. (1999). A novel four-node quadrilateral smoothing element for stress enhancement and error estimation. International Journal for Numerical Methods in Engineering, 44(10), 1527-1541.

Tessler, A., \& Spangler, J.L. (2003). A variational principle for reconstruction of elastic deformation of shear deformable plates and shells, NASA TM-2003-212445.

Tessler, A., \& Spangler, J.L. (2004). Inverse FEM for full-field reconstruction of elastic deformations in shear deformable plates and shells. In: Proceedings of 2nd European Work-shop on Structural Health Monitoring, Munich, Germany.

Tessler, A., \& Spangler, J. L. (2005). A least-squares variational method for full-field reconstruction of elastic deformations in shear-deformable plates and shells. Computer Methods in Applied Mechanics and Engineering, 194(2), 327-339.

Tessler, A. (2007). Structural Analysis Methods for Structural Health Management of Future Aerospace Vehicles. Key Engineering Materials, 347, 57-66. 
Tessler, A., Di Sciuva, M., \& Gherlone, M. (2009). A refined zigzag beam theory for composite and sandwich beams. Journal of Composite Materials, 43, 1051-1081.

Tessler, A., Di Sciuva, M., \& Gherlone, M. (2010). A consistent refinement of first-order shear deformation theory for laminated composite and sandwich plates using improved zigzag kinematics. Journal of Mechanics of Materials and Structures, 5(2), 341-367.

Tessler, A., Spangler, J. L., Gherlone, M., Mattone, M., \& Di Sciuva, M. (2011). Real-Time characterization of aerospace structures using on-board strain measurement technologies and inverse finite element method. In: Proceedings of the 8th International Workshop on Structural Health Monitoring, Stanford, CA.

Tessler, A., Spangler, J. L., Gherlone M., Mattone M., \& Di Sciuva, M. (2012). Deformed shape and stress reconstruction in plate and shell structures undergoing large displacements: application of inverse finite element method using fiber bragg grating strains. In: Proceedings of 10th World Congress on Computational Mechanics, Sao Paulo, Brazil.

Torkildsen, H. E., Grovlen, A., Skaugen, A., Wang, G., Jensen, A. E., Pran, K., \& Sagvolden, G. (2005). Development and applications of full-scale ship hull health monitoring systems for the Royal Norwegian Navy. Norwegian Defence Research Establishment Kjeller.

Van der Cammen, J. J. (2008). Fatigue prediction and response monitoring on a FPSO. TU Delft, Delft University of Technology.

Vazquez, S.L., Tessler, A., Quach, C.C., Cooper, E.G., Parks, J., \& Spangler J.L. (2005). Structural health monitoring using high-density fiber optic strain sensor and inverse finite element methods, NASA TM-2005-213761.

Wu, M., \& Hermundstad, O. A. (2002). Time-domain simulation of wave-induced nonlinear motions and loads and its applications in ship design. Marine Structures, 15(6), 561-597.

Zhu, B., \& Frangopol, D. M. (2013). Incorporation of structural health monitoring data on load effects in the reliability and redundancy assessment of ship cross-sections using Bayesian updating. Structural Health Monitoring, 12(4), 377-392. 


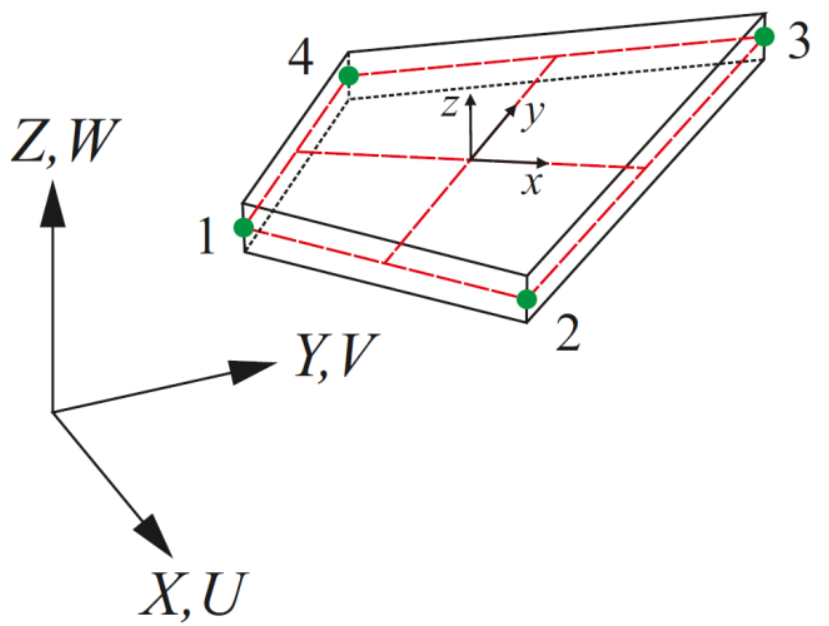

(a)

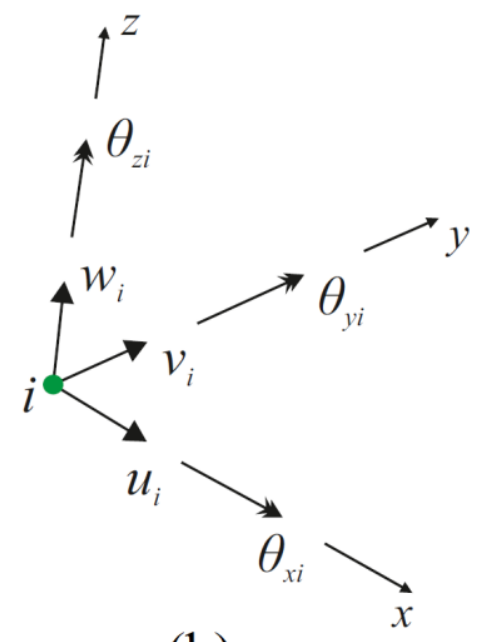

(b)

Fig. 1. (a) Four-node quadrilateral inverse shell element, iQS4, depicted within global $(X, Y, Z)$ and local $(x, y, z)$ frames of reference; (b) Nodal degrees-of-freedom corresponding to local (element) coordinates $(x, y, z)$.

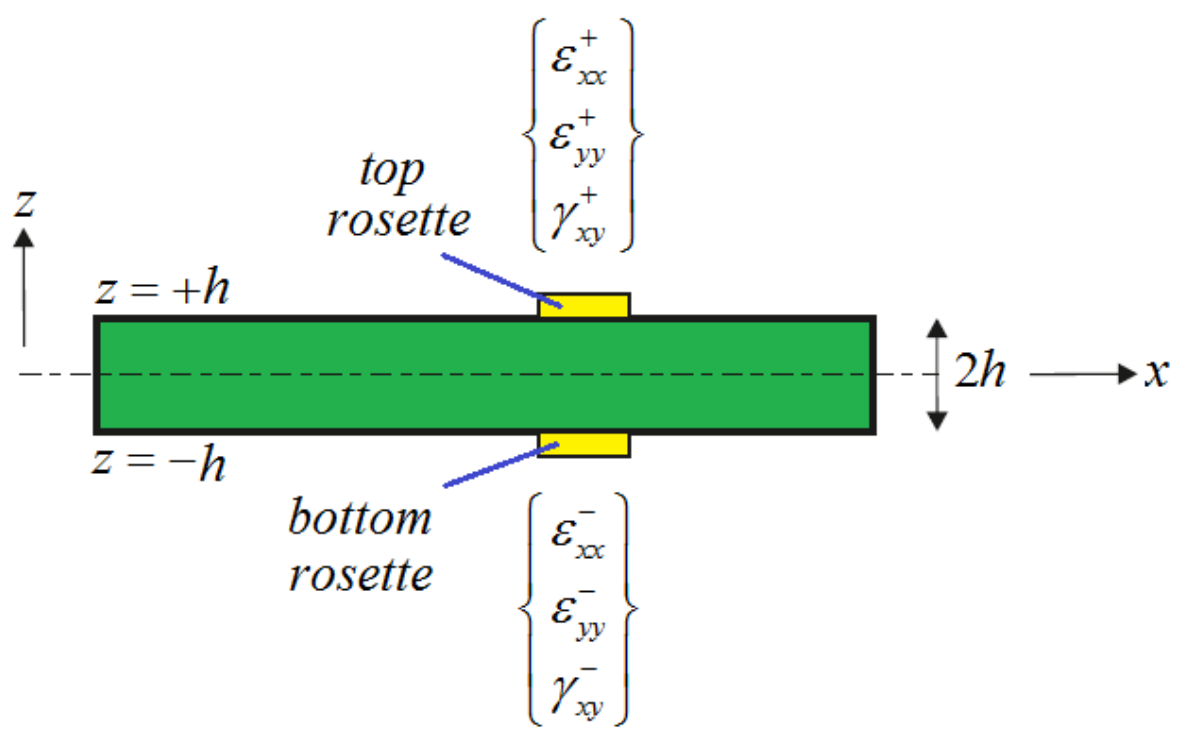

Fig. 2. Discrete surface strains measured by strain rosettes within iQS4 element at

$$
\mathbf{x}_{i}=\left(x_{i}, y_{i}, \pm h\right) \text { locations. }
$$




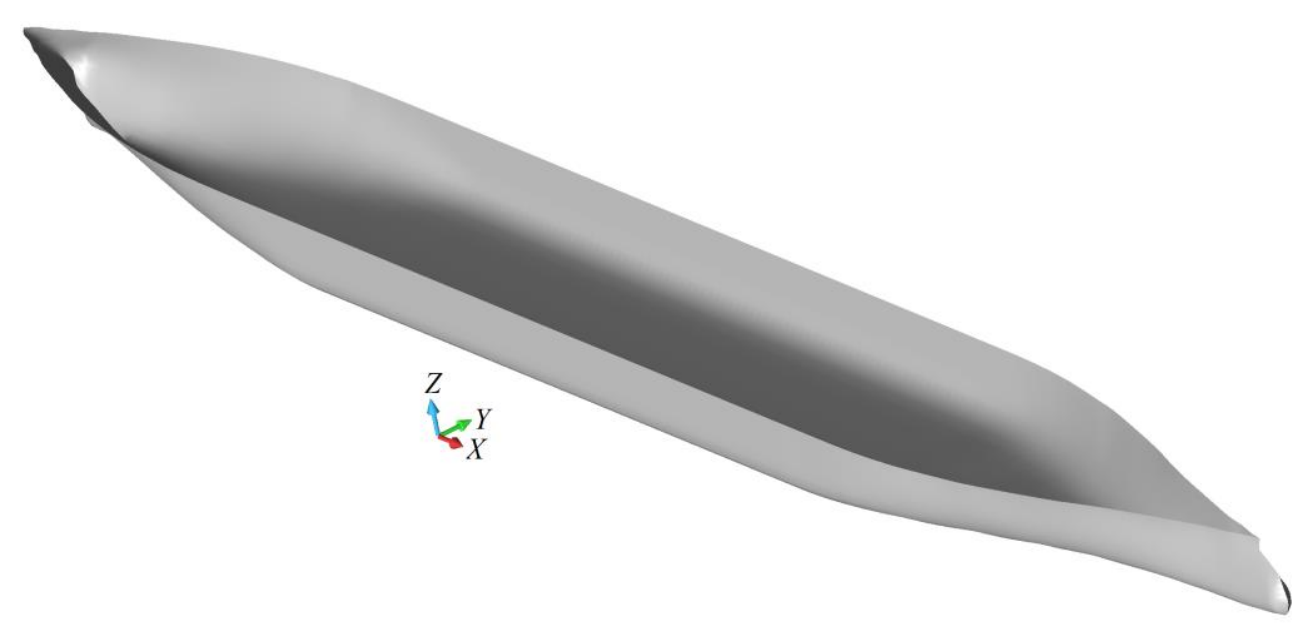

Fig. 3. Isometric view of the hull surface below draft waterline.

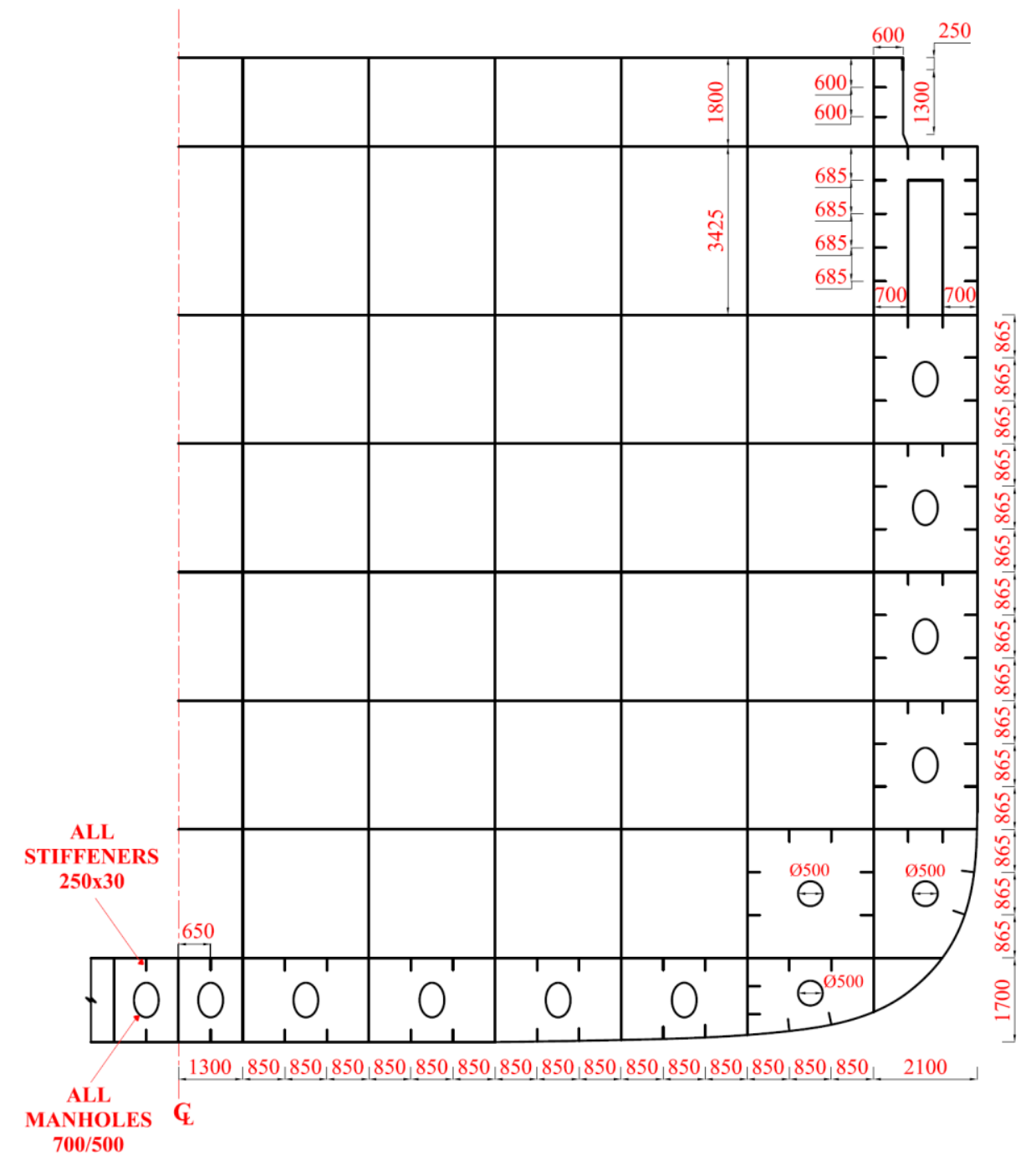

Fig. 4. Mid-ship section of the Panamax containership. 


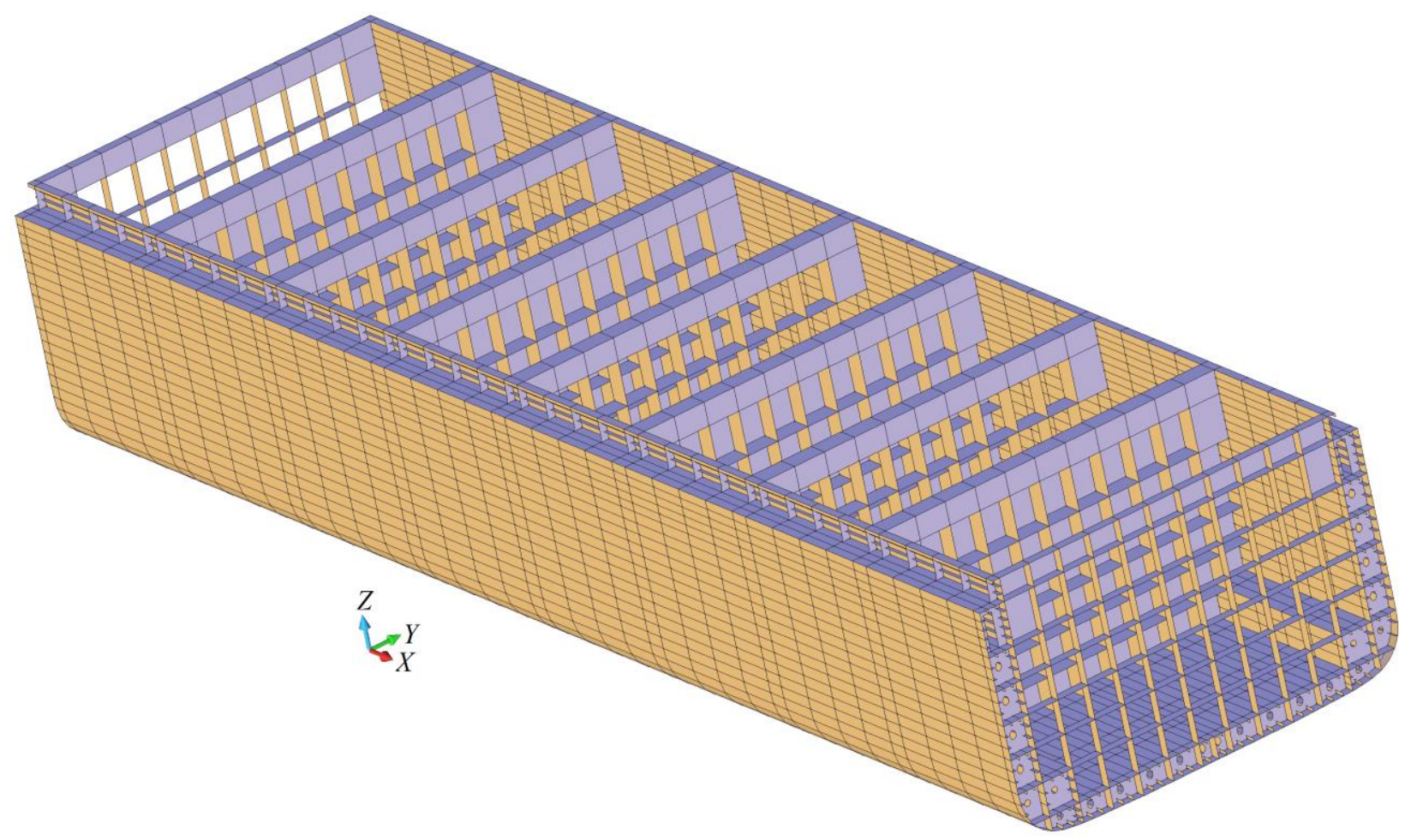

Fig. 5. Parallel mid-body of the Panamax containership.

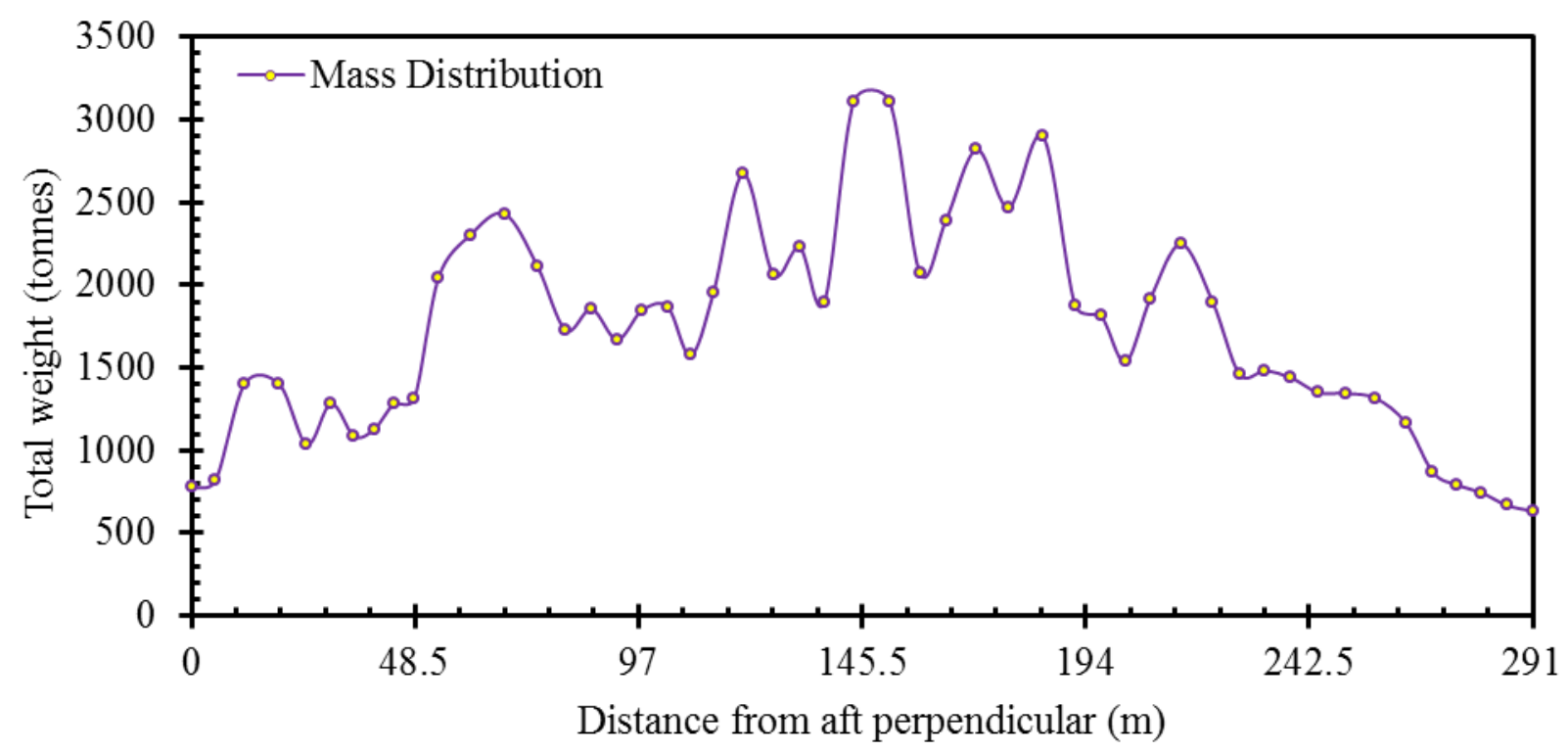

Fig. 6. Total weight distribution of the containership. 


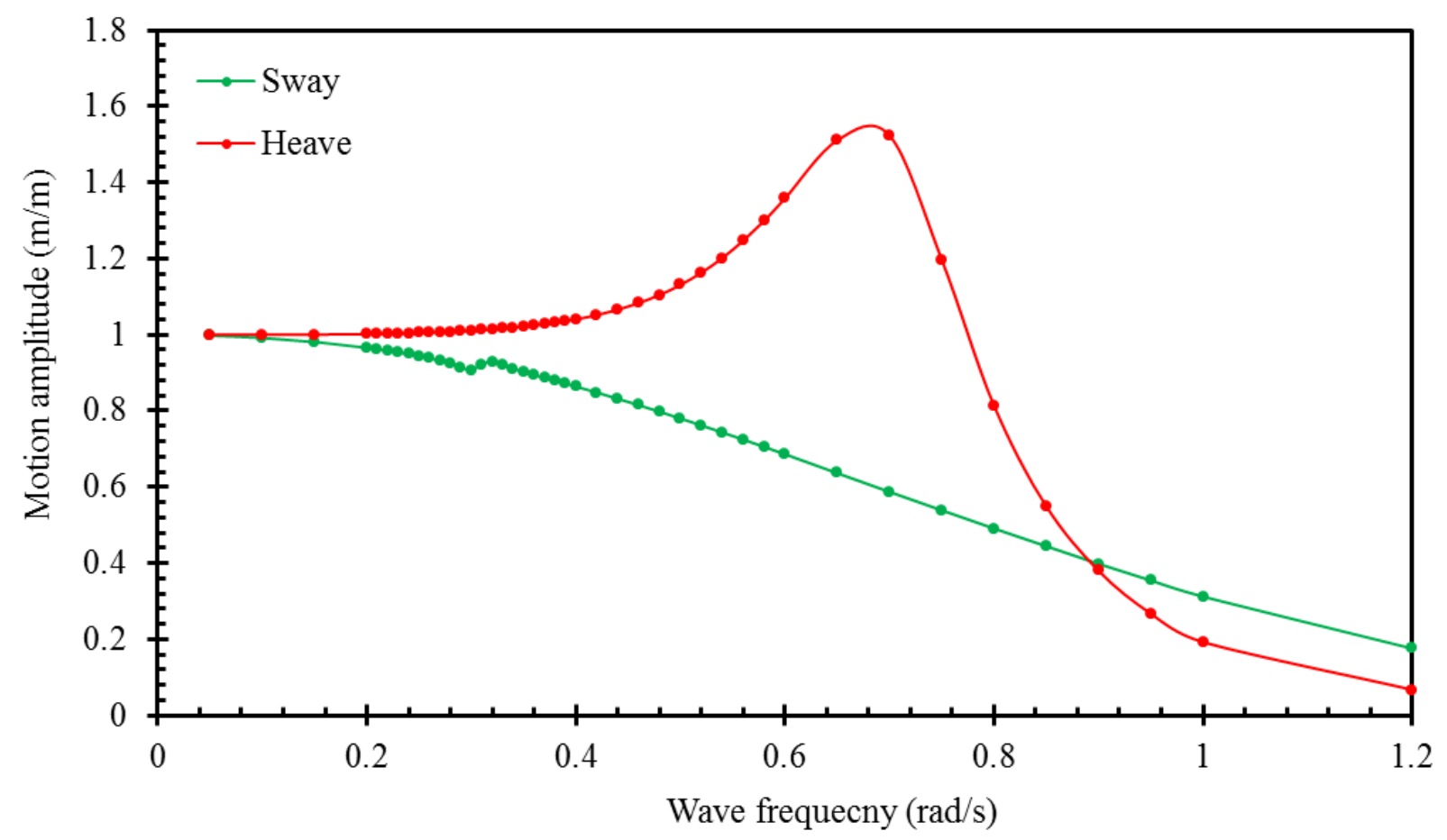

Fig. 7. Sway and heave motion amplitudes.

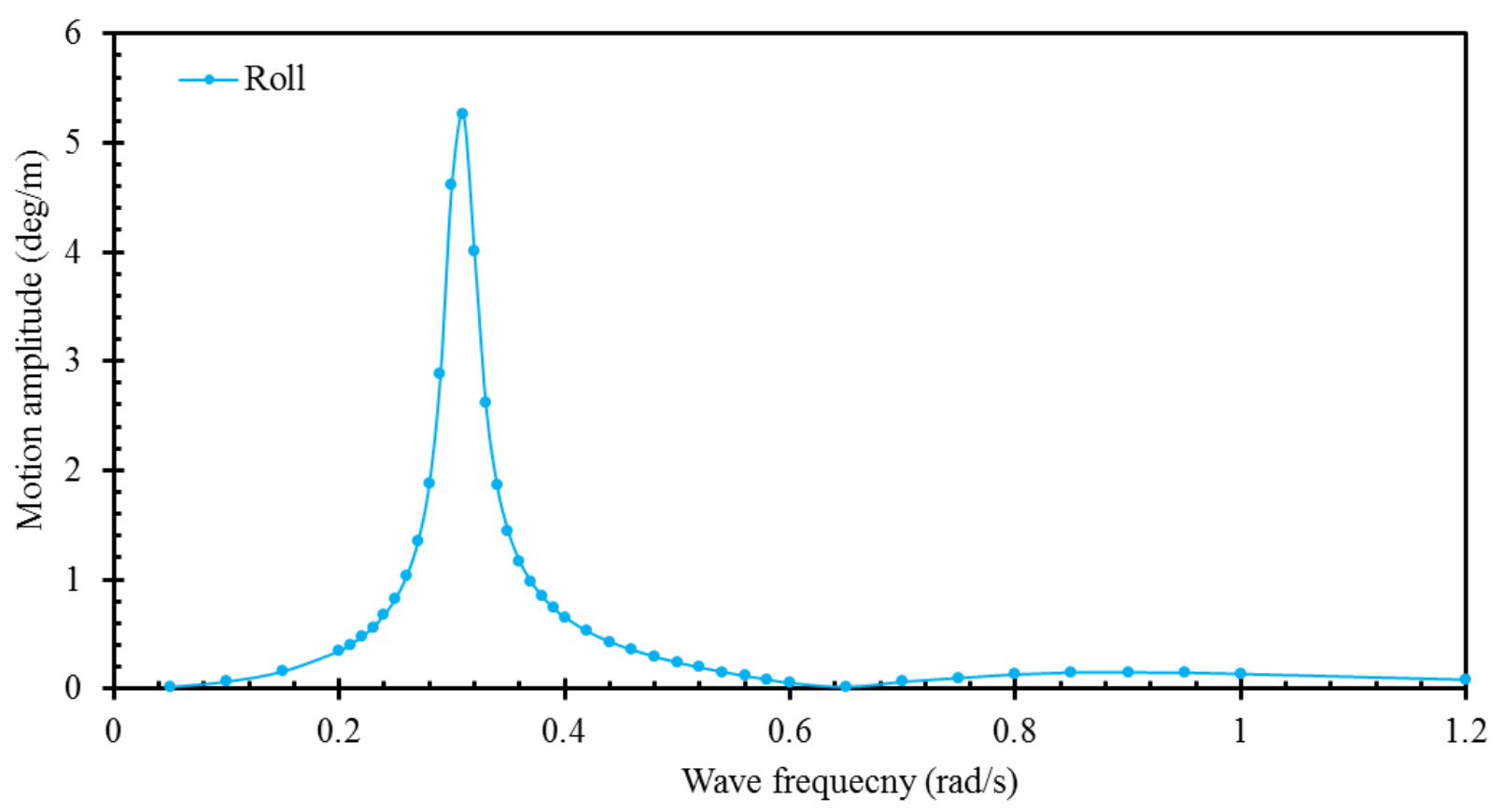

Fig. 8. Roll motion amplitudes. 


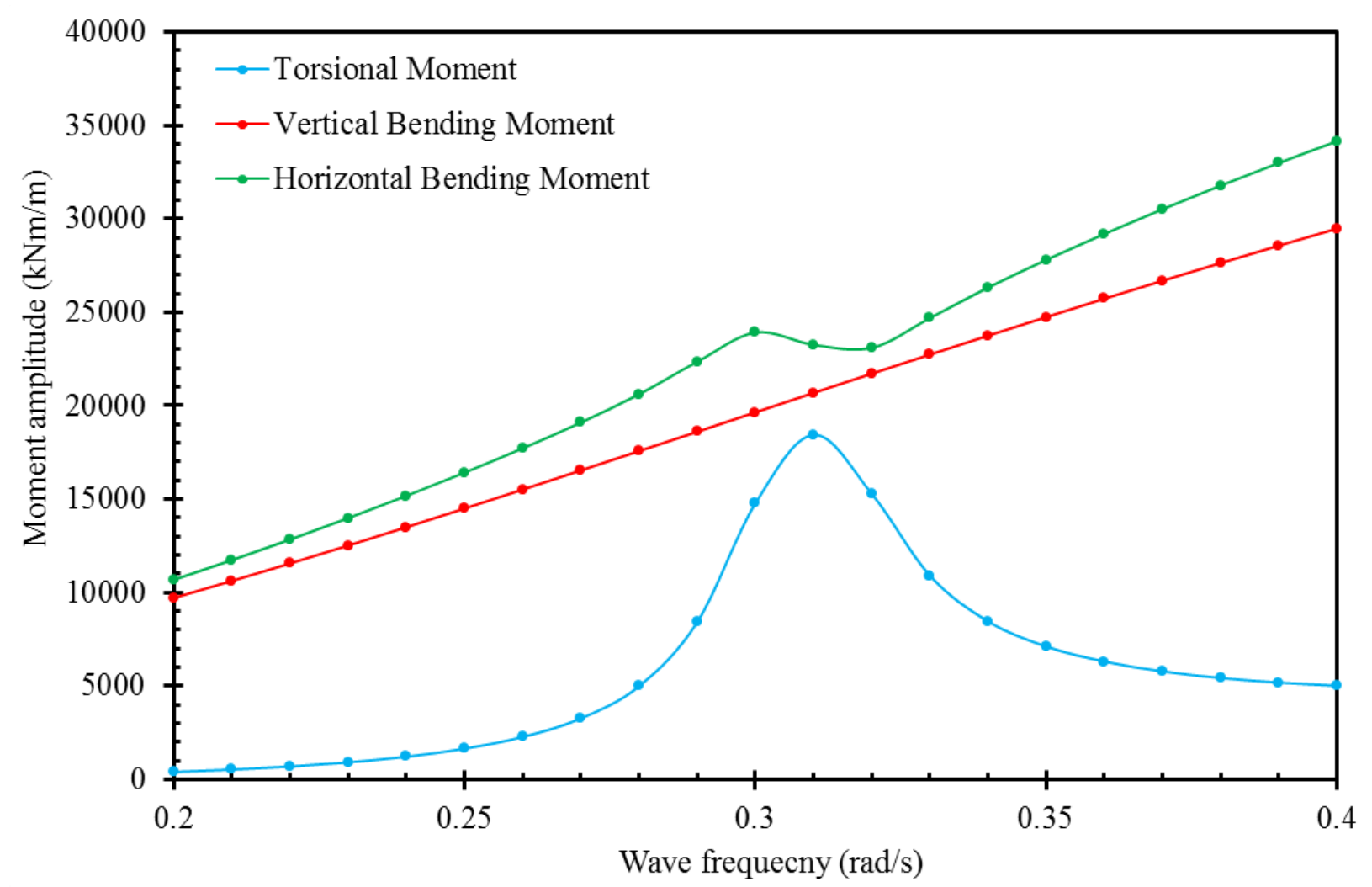

Fig. 9. Wave vertical and horizontal bending and torsional moments.

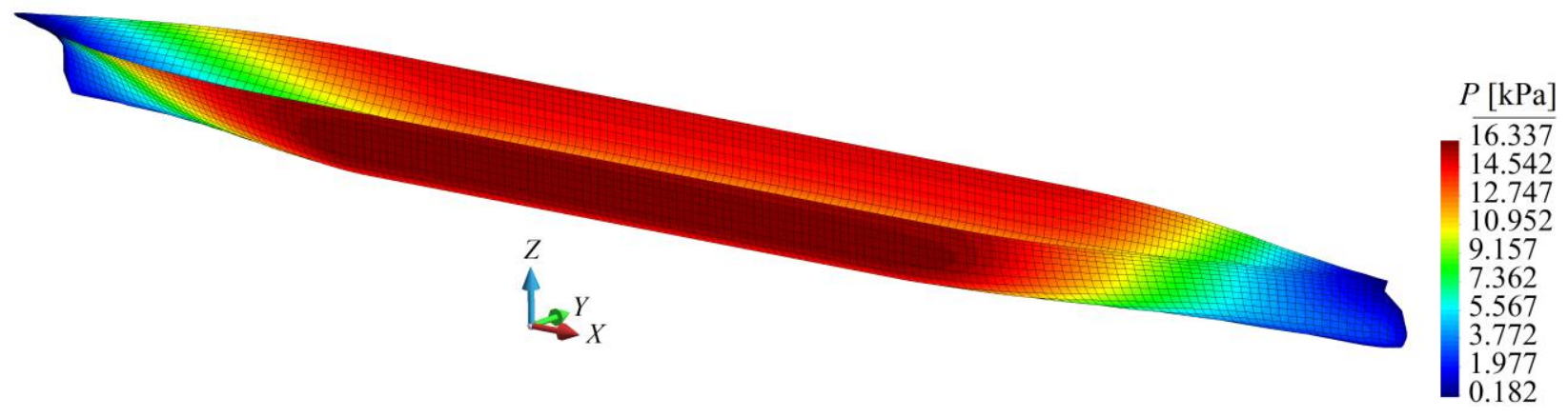

Fig. 10. Contour plot of total hydrodynamic pressure $P$ at wave frequency of $0.31 \mathrm{rad} / \mathrm{s}$. 


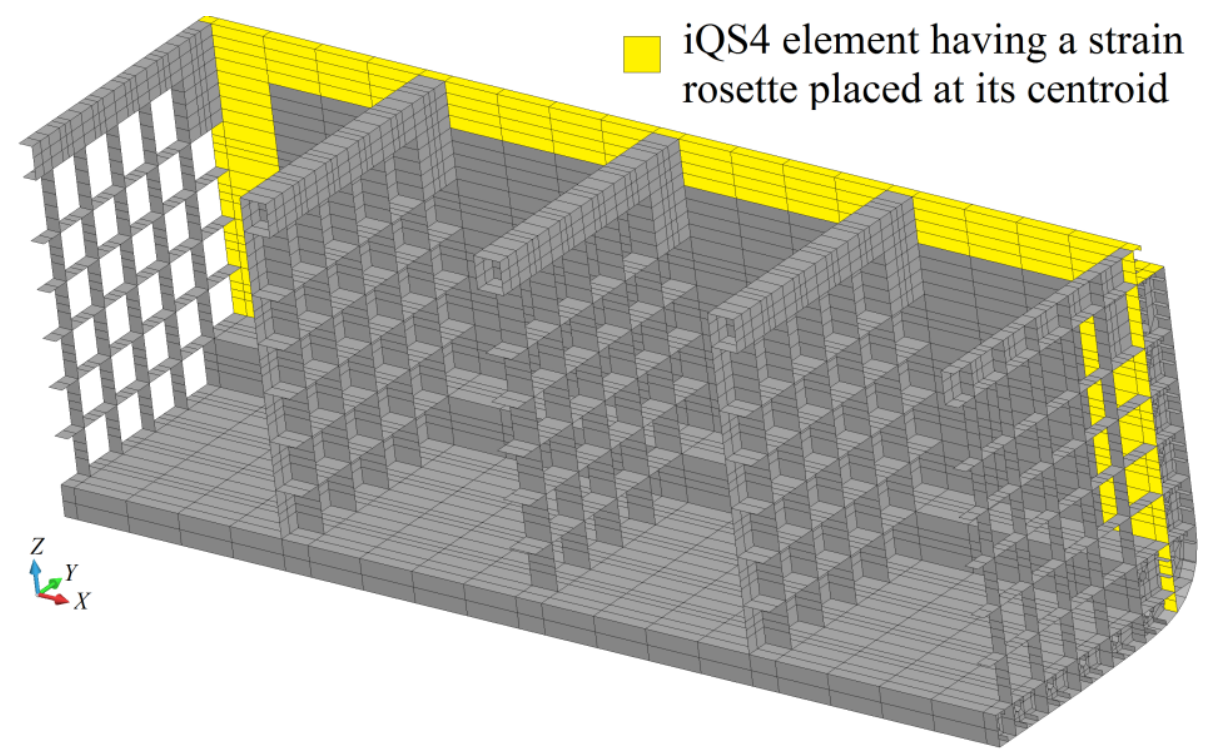

Fig. 11. Discretization of one-fourth of parallel mid-body using 15,318 iQS4 elements with topand bottom-surface strain rosettes located within 327 selected elements: Isometric view I.

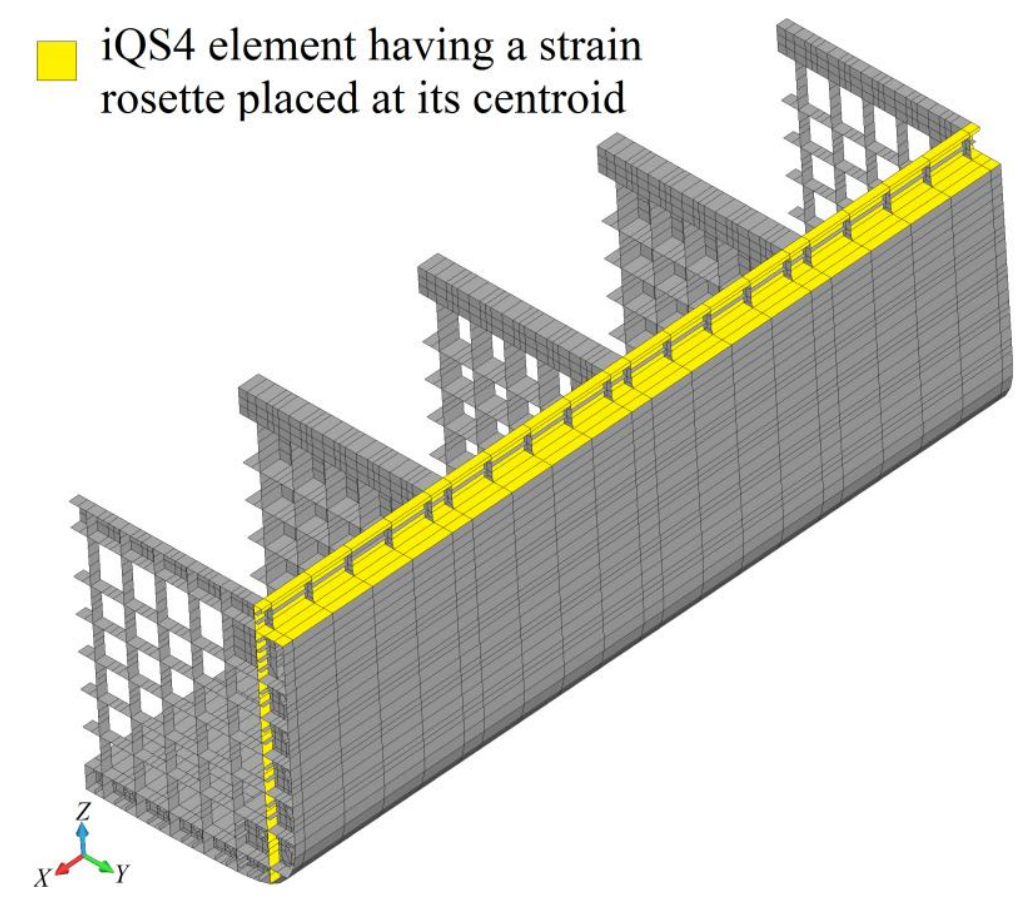

Fig. 12. Discretization of one-fourth of parallel mid-body using 15,318 iQS4 elements with topand bottom-surface strain rosettes located within 327 selected elements: Isometric view II. 


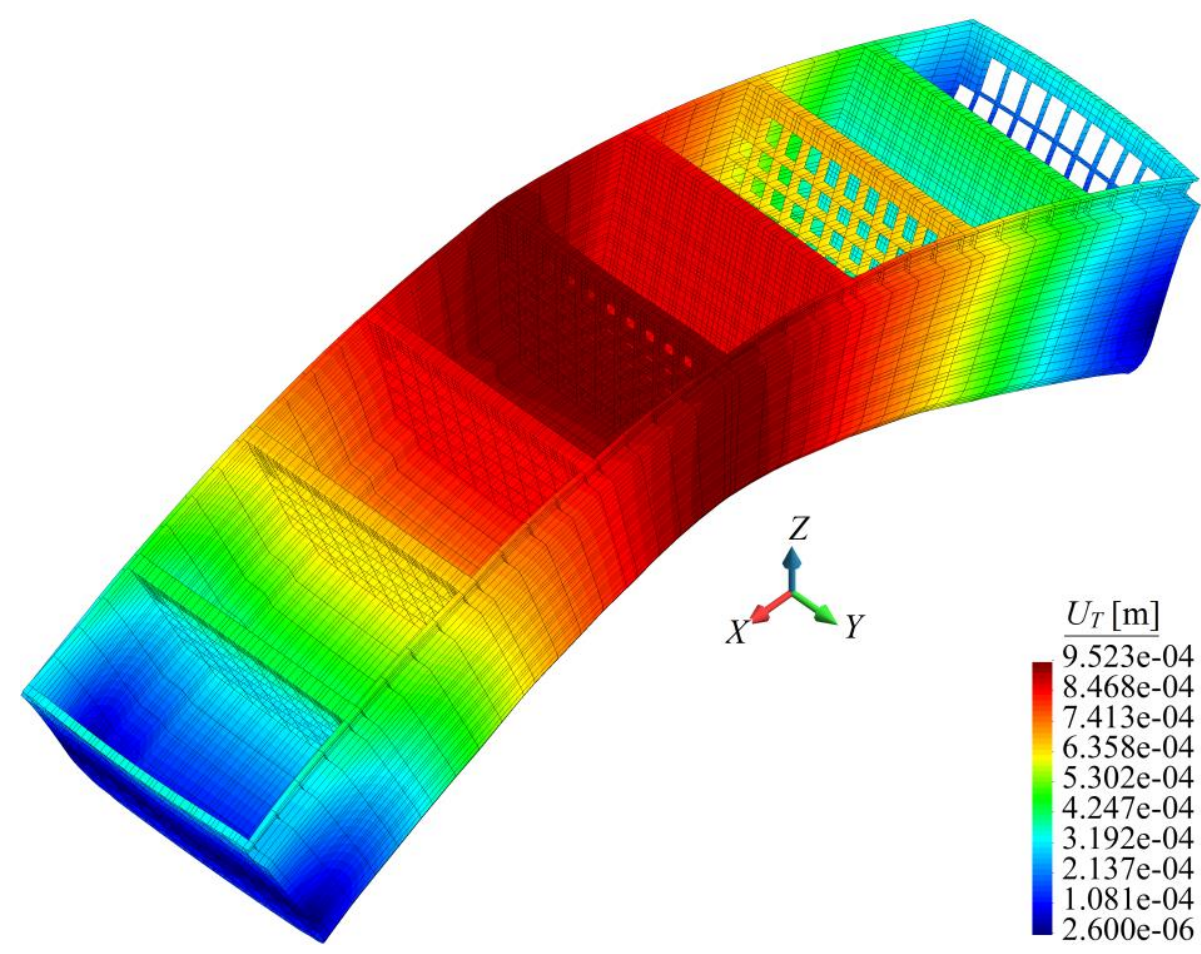

Fig. 13. Contour plot of $U_{T}$ displacement for parallel mid-body modelled in Figs. 11 and 12.

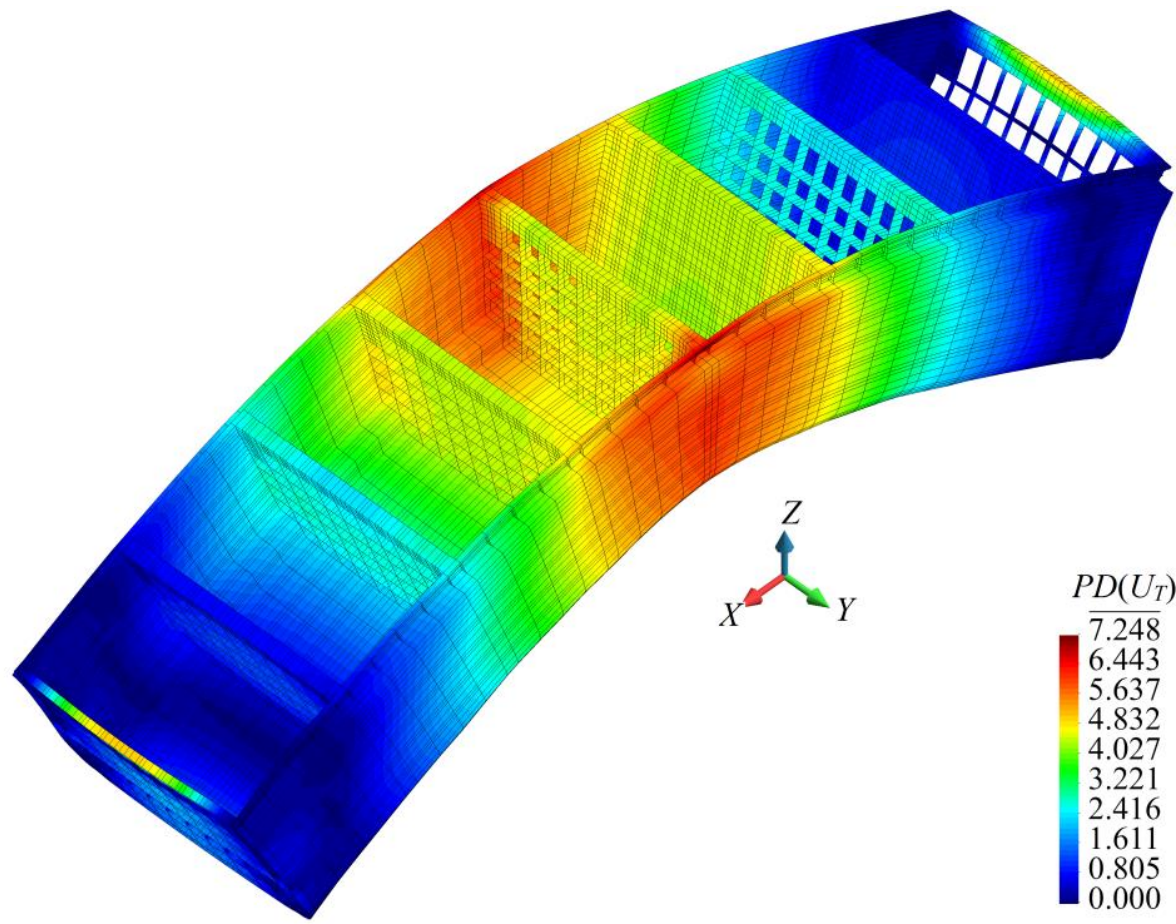

Fig. 14. Contour plot of $P D\left(U_{T}\right)$ percent difference for parallel mid-body modelled in Figs. 11 and 12. 


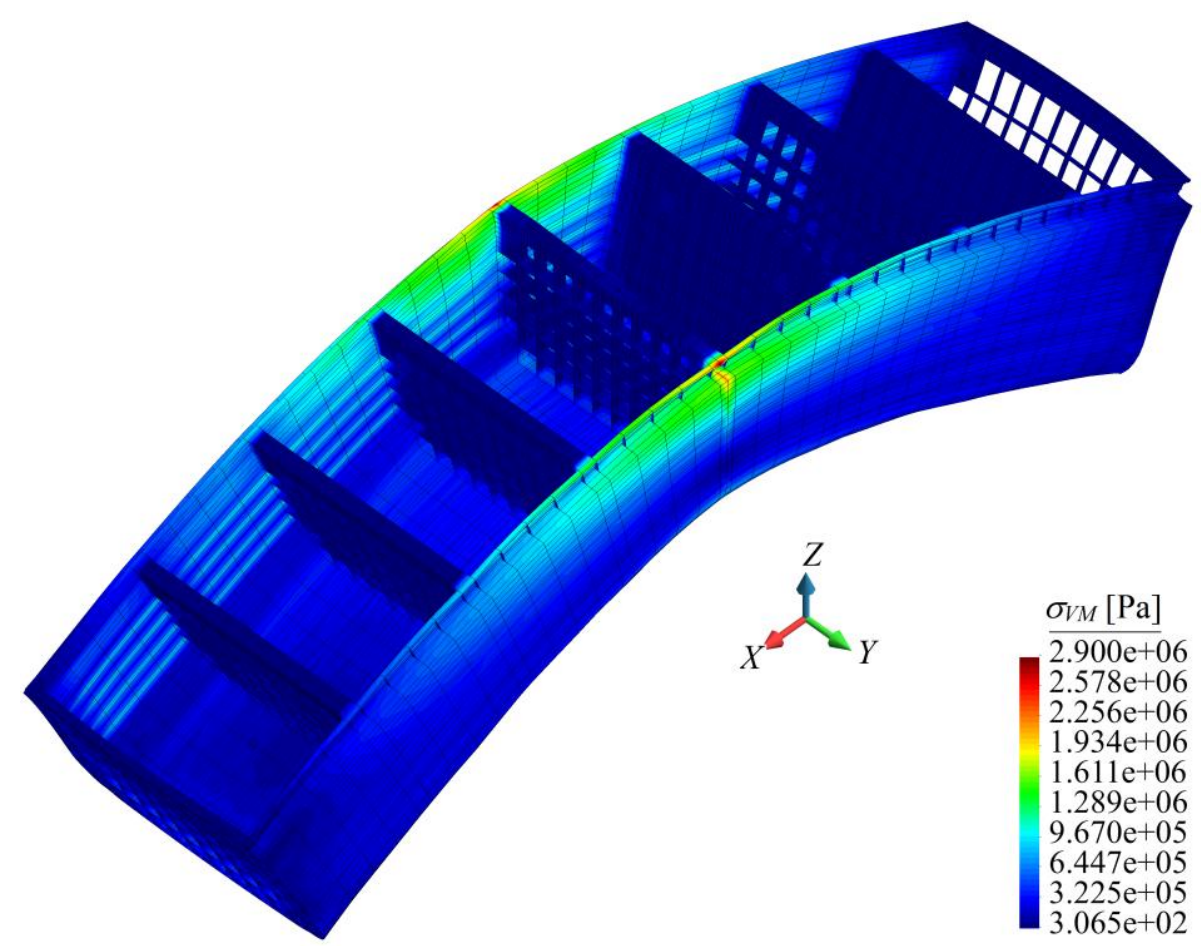

Fig. 15. Contour plot of $\sigma_{V M}$ stress for parallel mid-body modelled in Figs. 11 and 12.

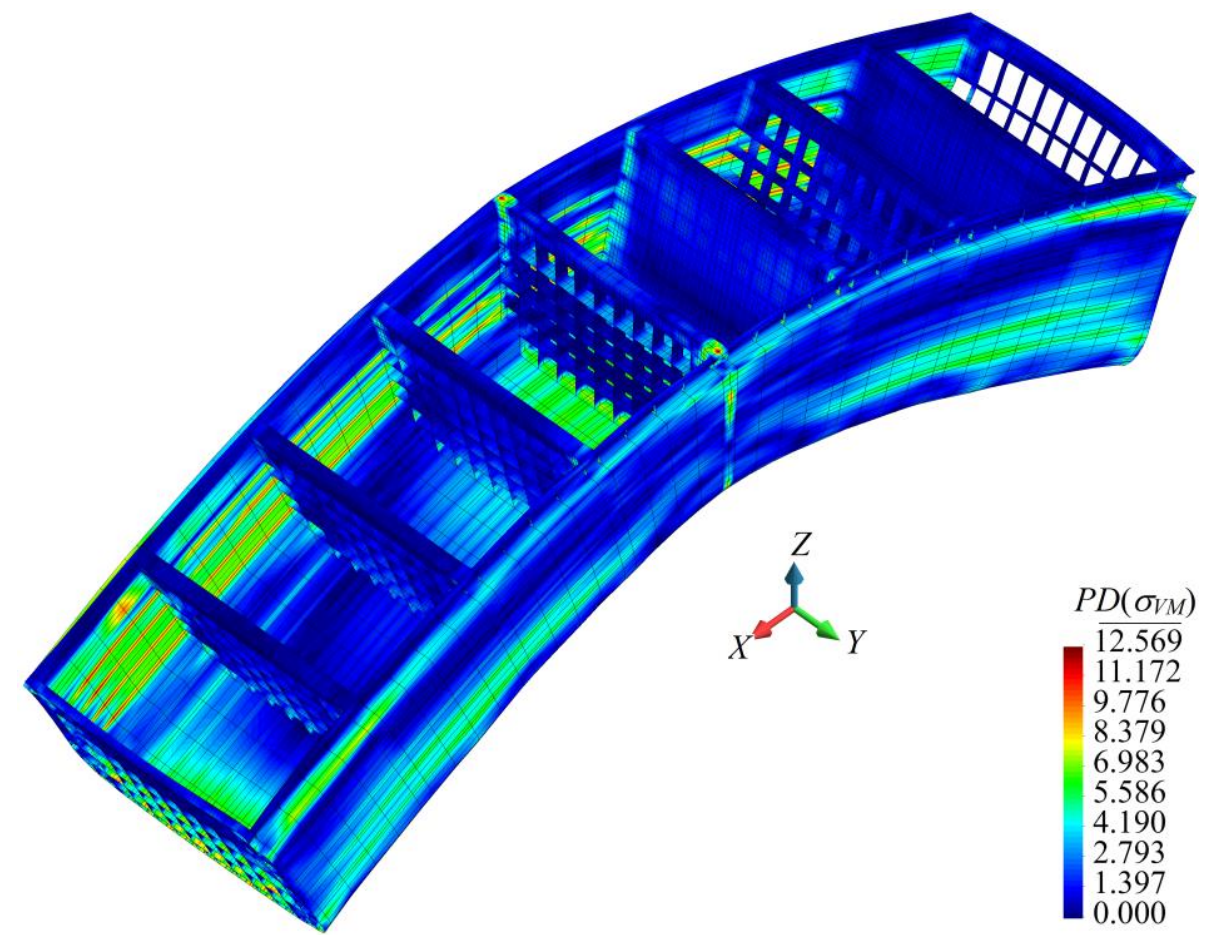

Fig. 16. Contour plot of $P D\left(\sigma_{V M}\right)$ percent difference for parallel mid-body modelled in Figs. 11 and 12 . 


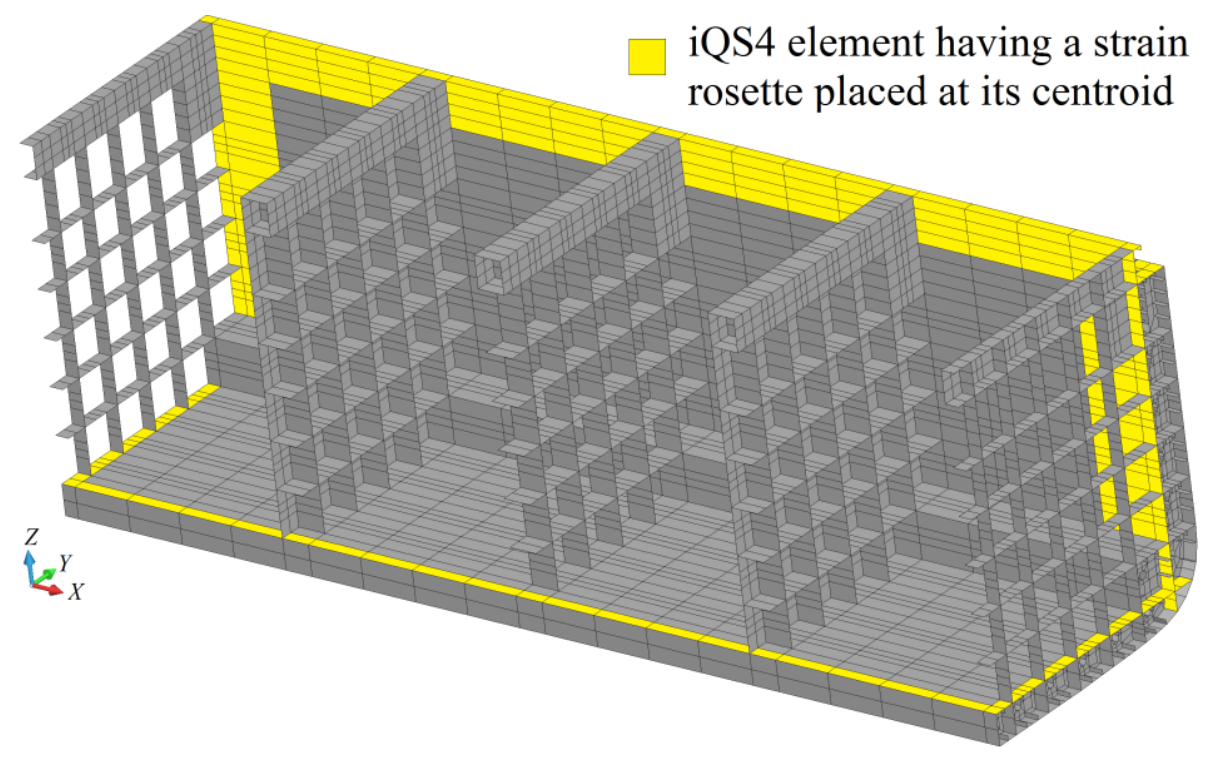

Fig. 17. Discretization of one-fourth of parallel mid-body using 15,318 iQS4 elements with topand bottom-surface strain rosettes located within 413 selected elements: Isometric view I.

iQS4 element having a strain rosette placed at its centroid

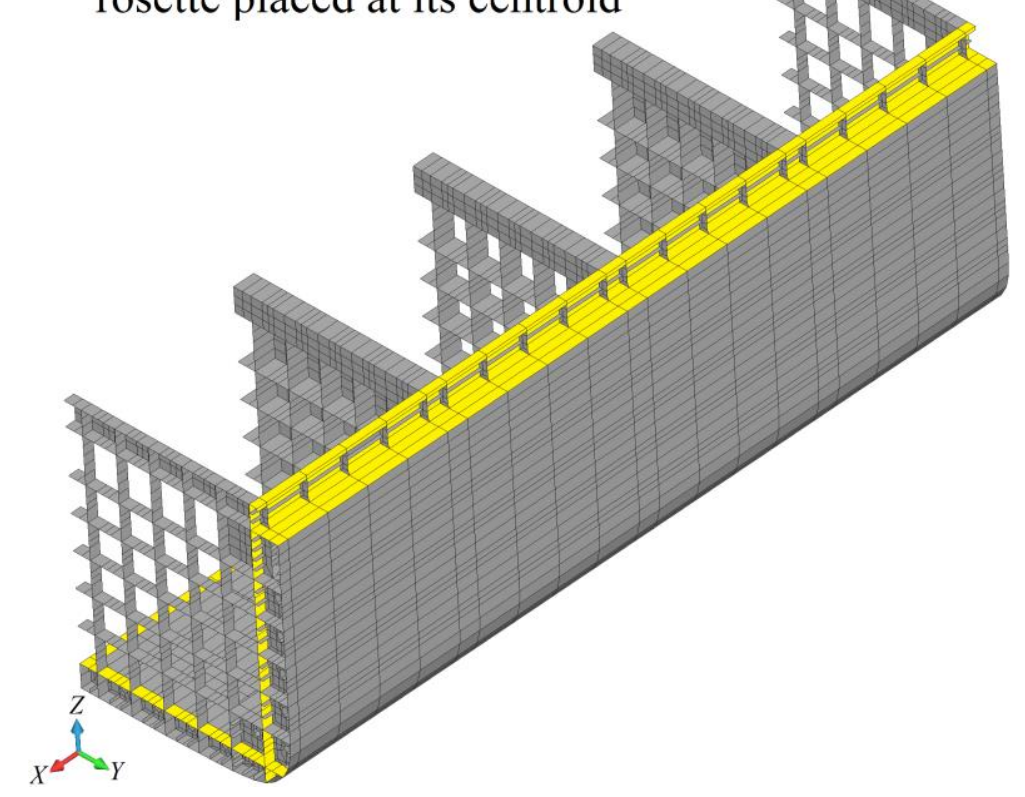

Fig. 18. Discretization of one-fourth of parallel mid-body using 15,318 iQS4 elements with topand bottom-surface strain rosettes located within 413 selected elements: Isometric view II. 


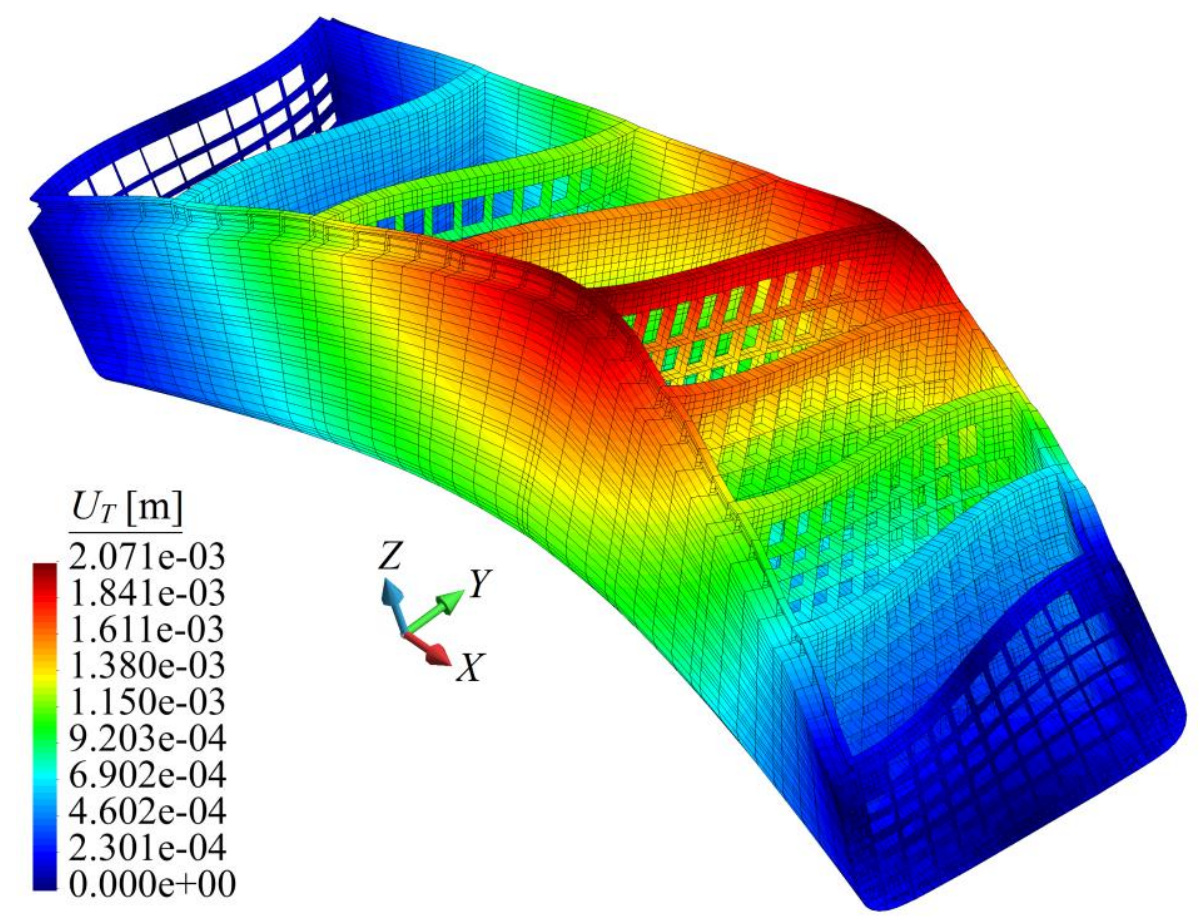

Fig. 19. Contour plot of $U_{T}$ displacement for parallel mid-body modelled in Figs. 17 and 18.

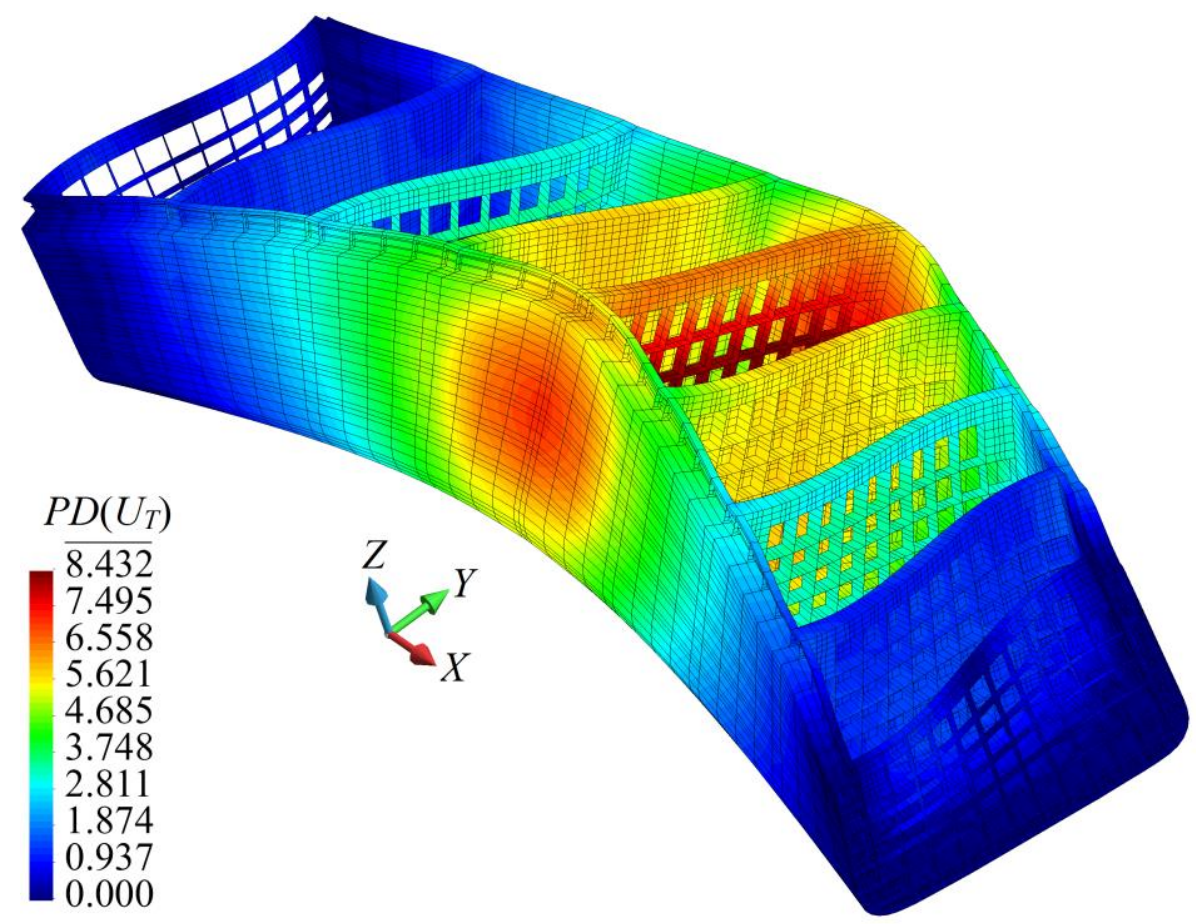

Fig. 20. Contour plot of $P D\left(U_{T}\right)$ percent difference for parallel mid-body modelled in Figs. 17 and 18. 


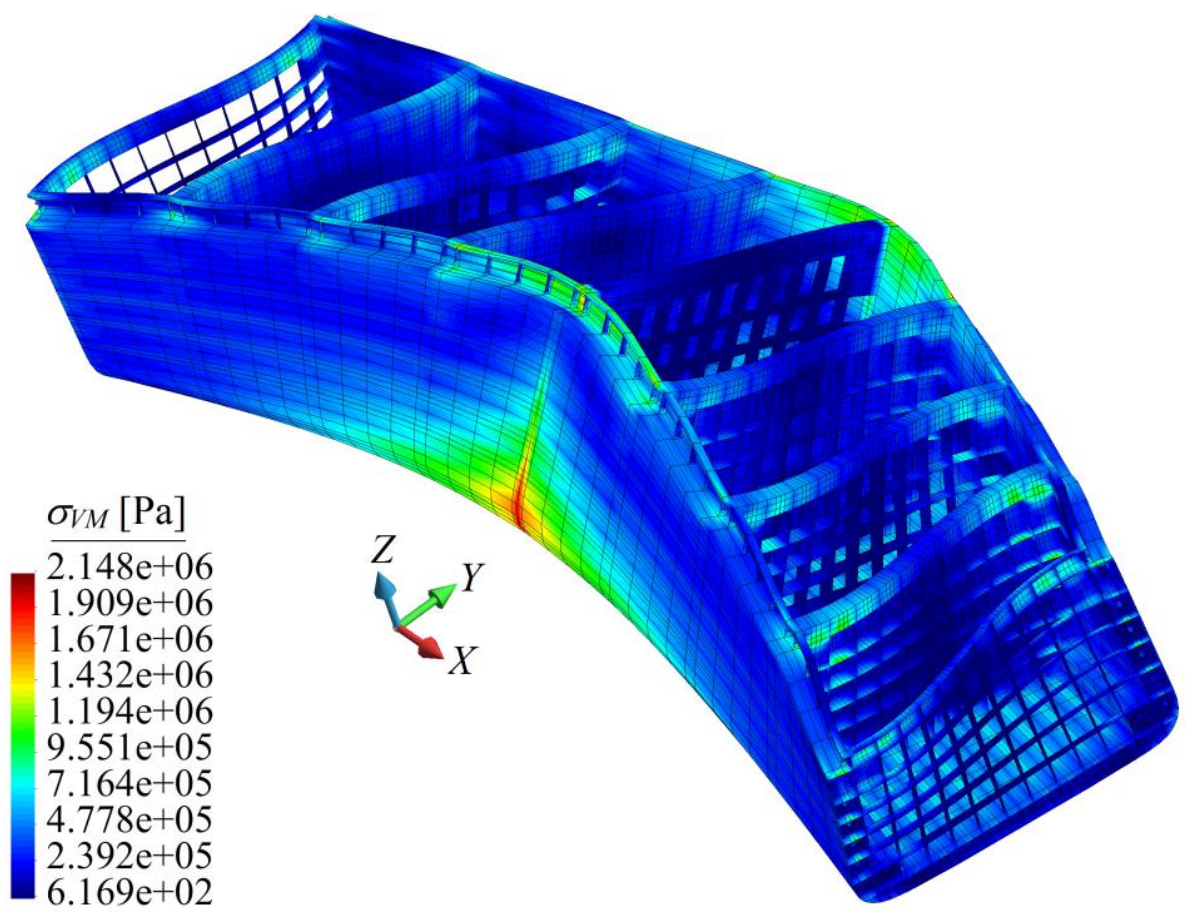

Fig. 21. Contour plot of $\sigma_{V M}$ stress for parallel mid-body modelled in Figs. 17 and 18.

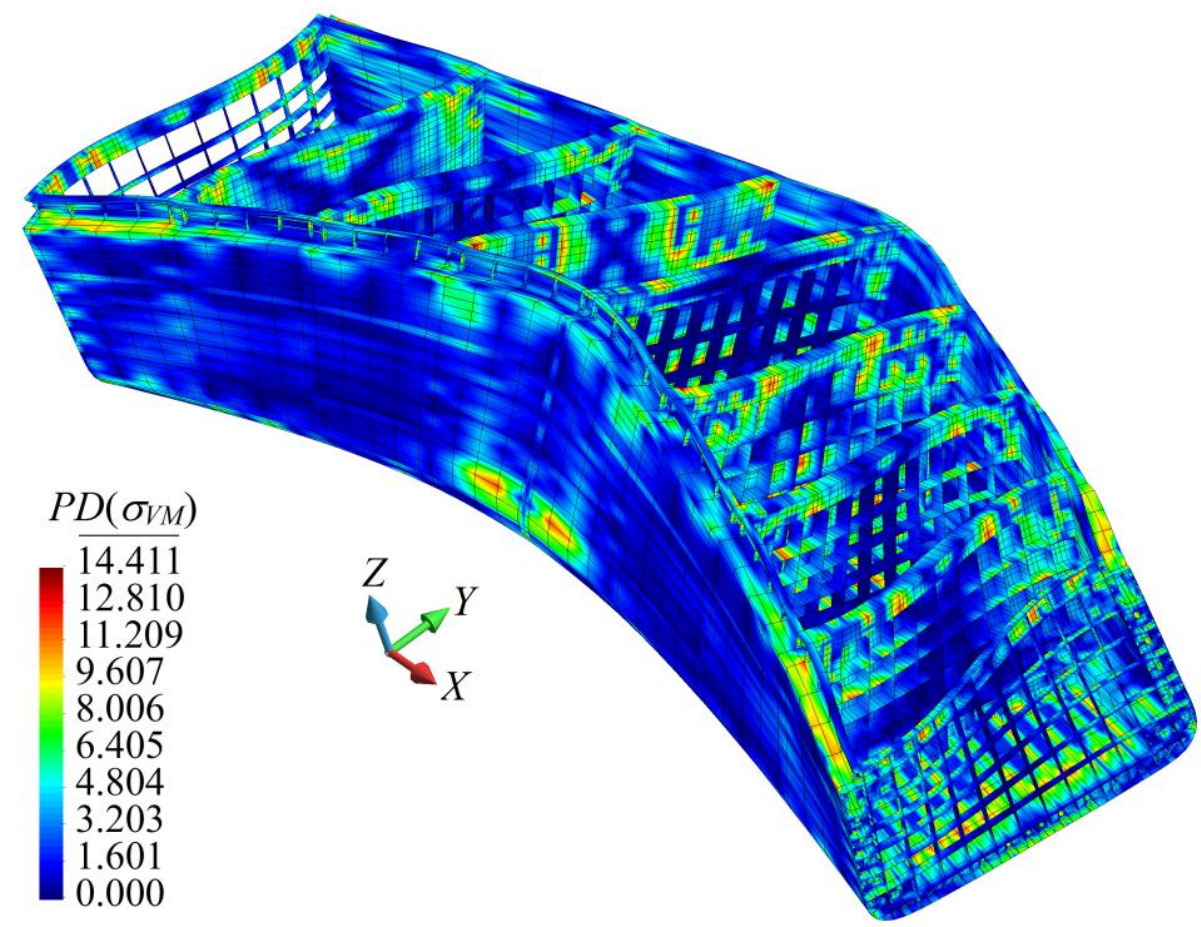

Fig. 22. Contour plot of $P D\left(\sigma_{V M}\right)$ percent difference for parallel mid-body modelled in Figs. 17 and 18. 


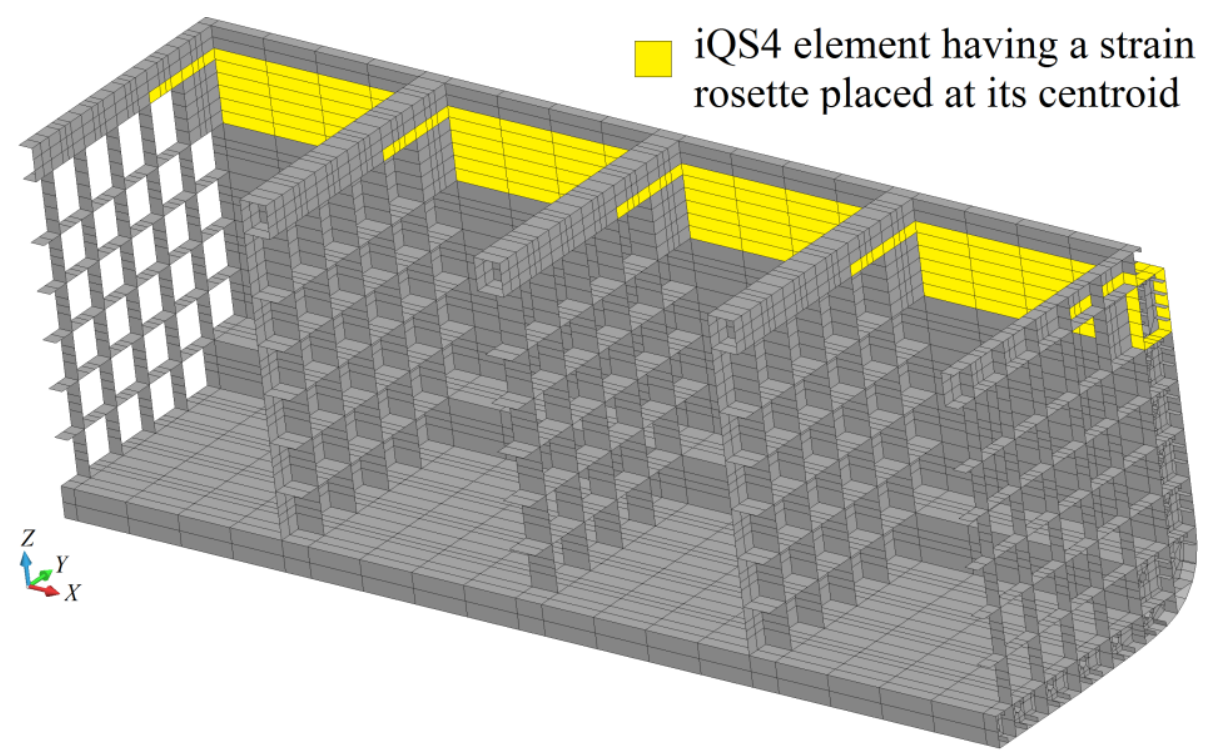

Fig. 23. Discretization of one-fourth of parallel mid-body using 15,318 iQS4 elements with topand bottom-surface strain rosettes located within 442 selected elements: Isometric view I.

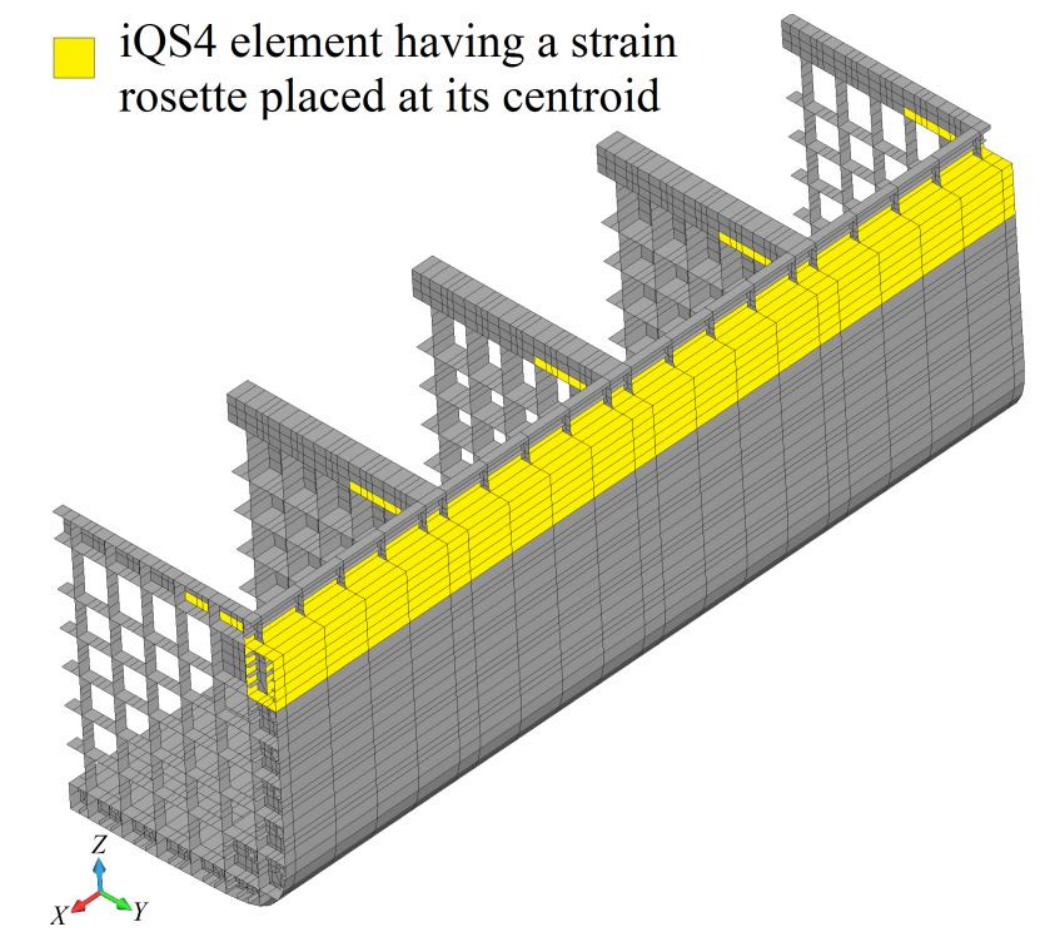

Fig. 24. Discretization of one-fourth of parallel mid-body using 15,318 iQS4 elements with topand bottom-surface strain rosettes located within 442 selected elements: Isometric view II. 


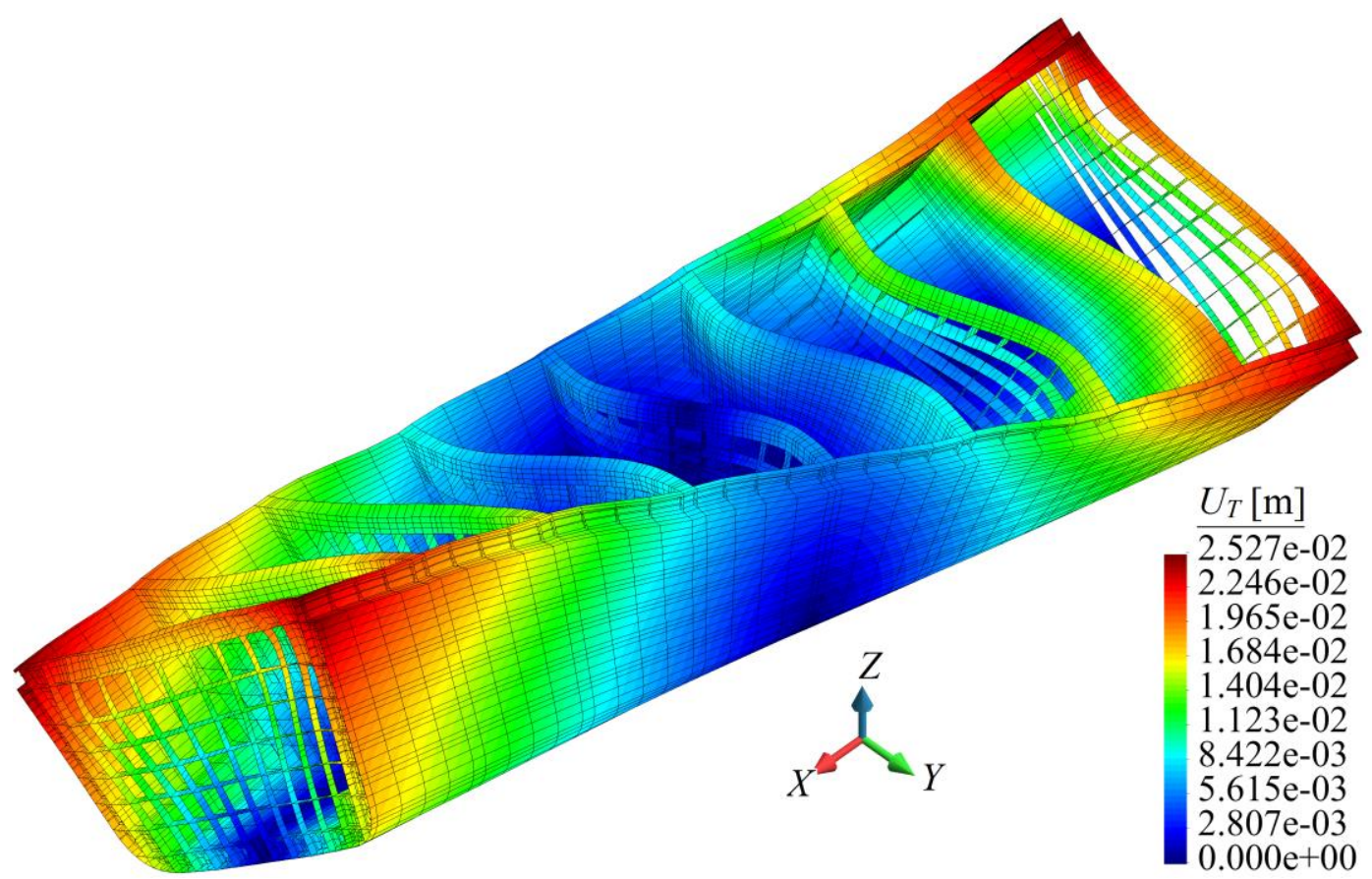

Fig. 25. Contour plot of $U_{T}$ displacement for parallel mid-body modelled in Figs. 23 and 24.

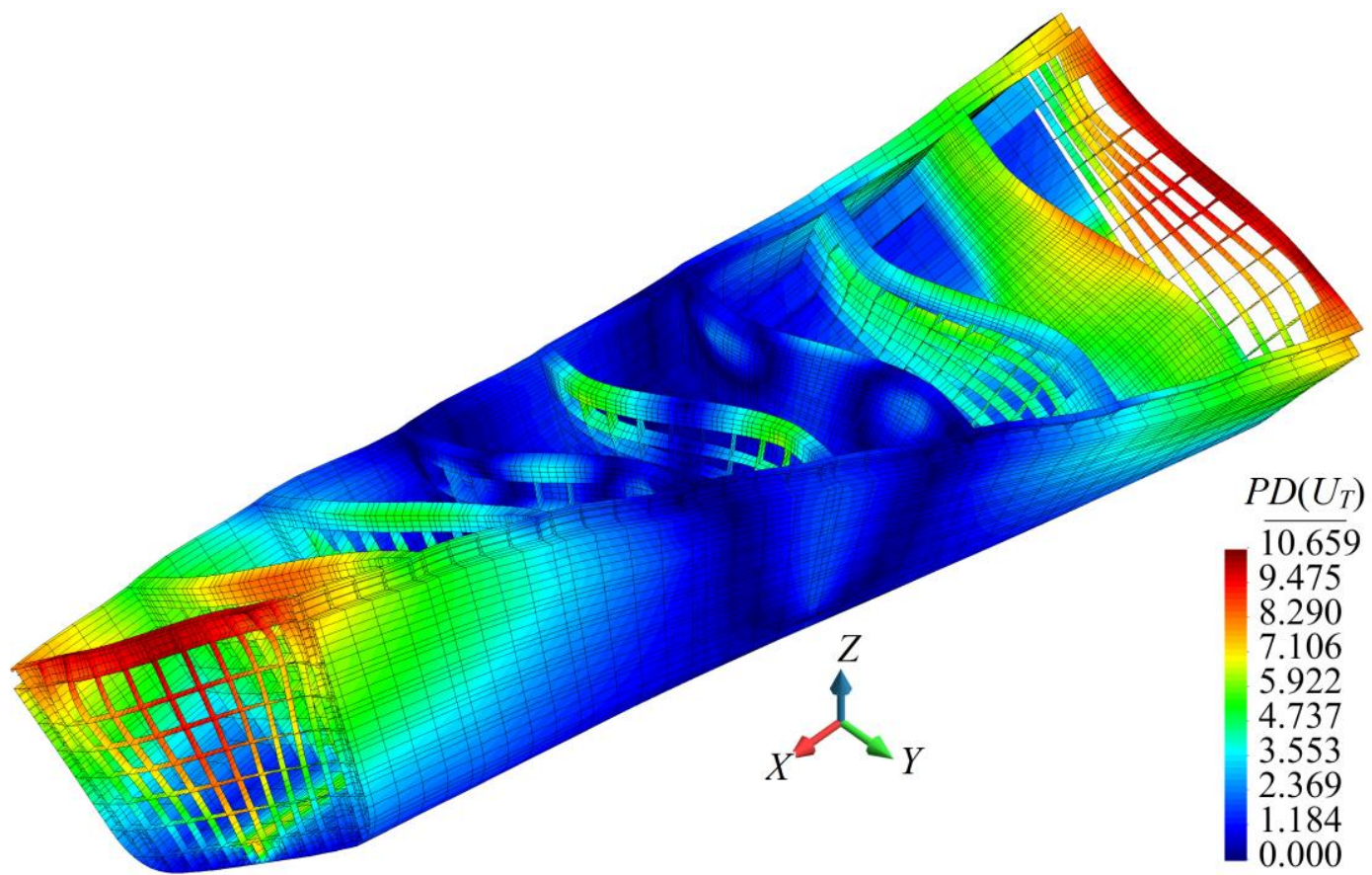

Fig. 26. Contour plot of $P D\left(U_{T}\right)$ percent difference for parallel mid-body modelled in Figs. 23 and 24. 


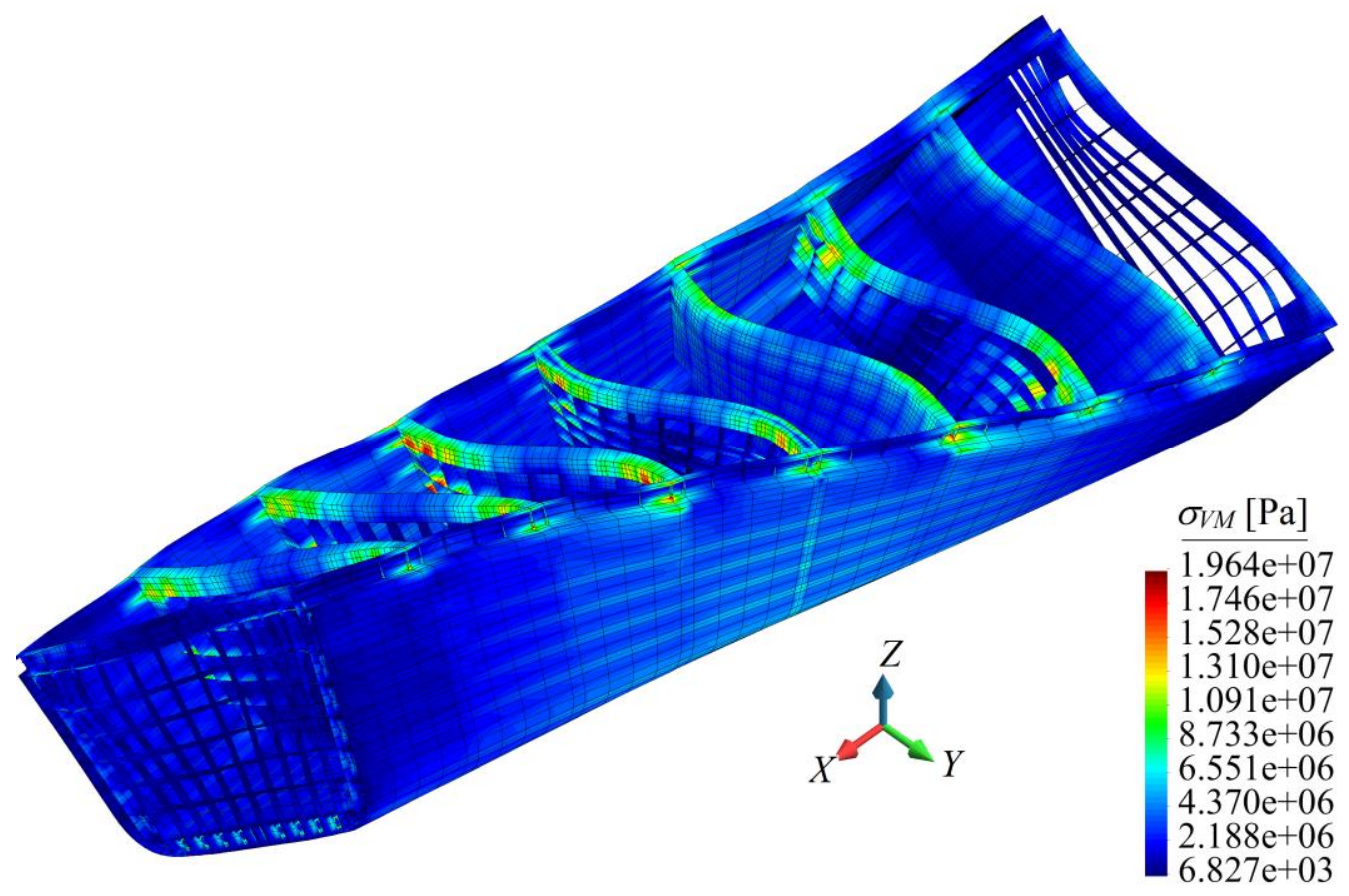

Fig. 27. Contour plot of $\sigma_{V M}$ stress for parallel mid-body modelled in Figs. 23 and 24.

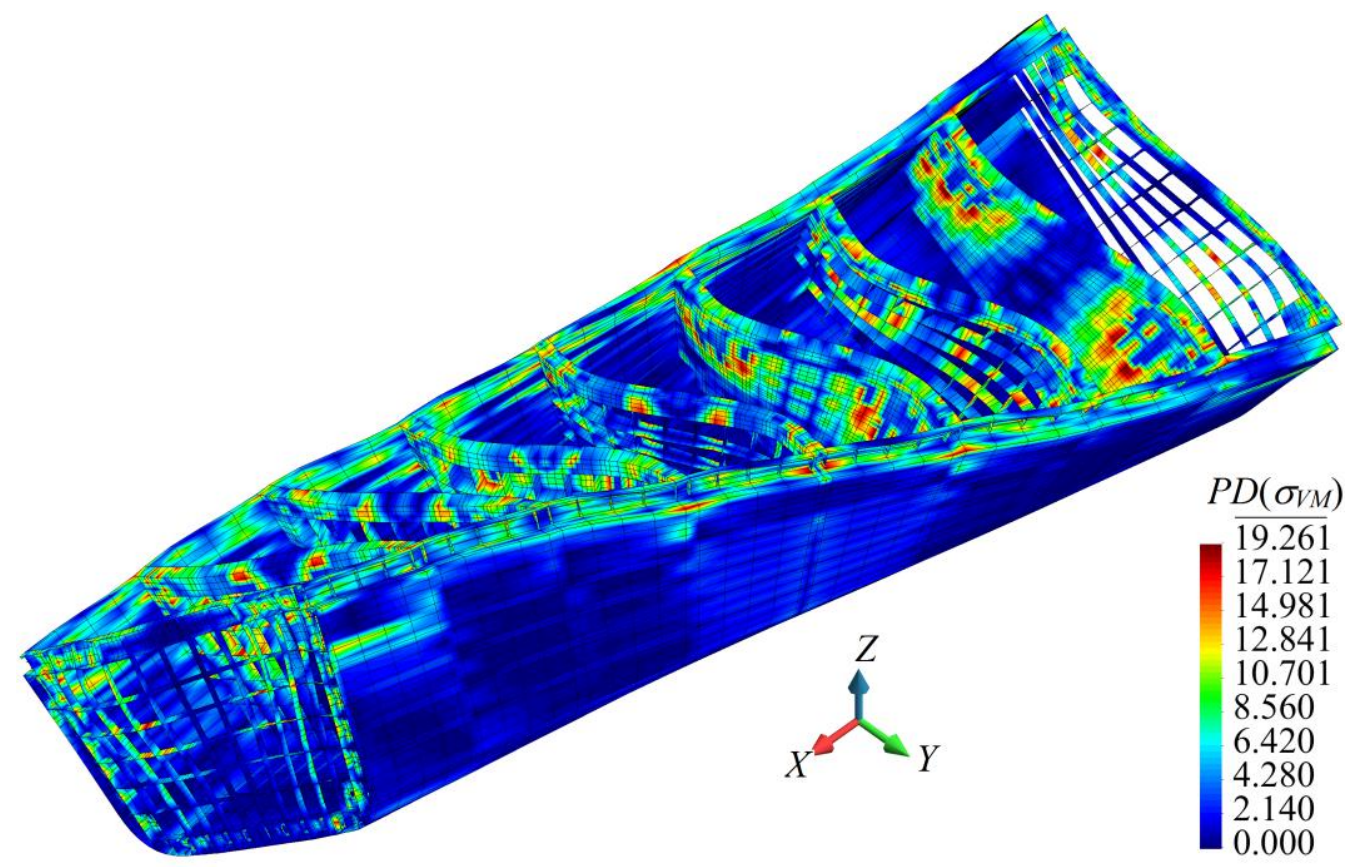

Fig. 28. Contour plot of $P D\left(\sigma_{V M}\right)$ percent difference for parallel mid-body modelled in Figs. 23 and 24. 
Table 1. General particulars of Panamax containership.

\begin{tabular}{lll}
\hline General particular & Value & Unit \\
\hline Length between perpendiculars & 291 & $\mathrm{~m}$ \\
Breadth (moulded) & 32.3 & $\mathrm{~m}$ \\
Depth (moulded) & 19.9 & $\mathrm{~m}$ \\
Design draft (moulded) & 12.1 & $\mathrm{~m}$ \\
Block coefficient & 0.73 & $\mathrm{~m}^{3} / \mathrm{m}^{3}$ \\
Displacement (at design draft) & 85190.5 & $\mathrm{tonnes}$ \\
Vertical centre of gravity (from baseline) & 12.1 & $\mathrm{~m}$ \\
Vertical centre of buoyancy (from baseline) & 6.4 & $\mathrm{~m}$ \\
Longitudinal centre of gravity (from aft perpendicular) & 141.2 & $\mathrm{~m}$ \\
Longitudinal centre of buoyancy (from aft perpendicular) & 141.2 & $\mathrm{~m}$ \\
Radius of gyration around $X$-axis & 10.834 & $\mathrm{~m}$ \\
Radius of gyration around $Y$-axis & 74.105 & $\mathrm{~m}$ \\
Radius of gyration around $Z$-axis & 74.105 & $\mathrm{~m}$ \\
Radius of gyration for roll-yaw product of inertia & 0 & $\mathrm{~m}$ \\
\hline
\end{tabular}

Table 2. Constraint boundary conditions.

\begin{tabular}{lll}
\hline Loading case & $X Z$-plane & YZ-plane \\
\hline Pure vertical bending case & Symmetry BC & Symmetry BC \\
Pure horizontal bending case & Anti-symmetry BC & Symmetry BC \\
Pure torsion case & Anti-symmetry BC & Anti-symmetry BC \\
\hline
\end{tabular}

